\title{
OBSERVATION OF HIGH CURRENT EFFECTS IN HIGH ENERGY LINEAR ACCELERATORS*
}

\author{
John T. Seeman \\ Stanford Linear Accelerator Center \\ Stanford University \\ Stanford, California, 94309
}

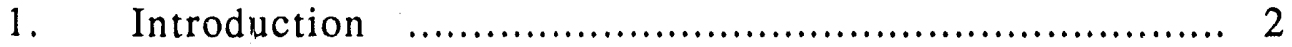

2. Longitudinal Effects ........................................ 2

2.1 Acceleration ......................................... 2

2.2. Energy spread ......................................... 3

2.3 Multibunch longitudinal effects.......................... 5

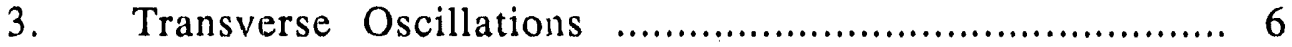

3.1 Equation of motion with transverse wakefields ............ 6

3.2 Transverse wakefield (BNS) damping ..................... 11

3.3 Analysis of oscillations ................................... 15

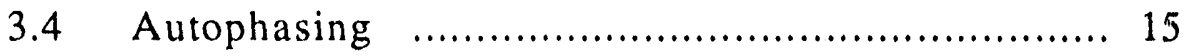

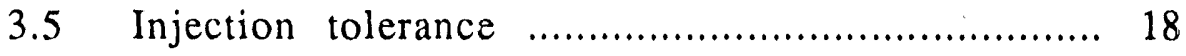

3.6 Transverse jitter ...................................... 18

4. Transverse Emittance Effects at High Currents ..................... 24

4.1 General emittance reduction techniques ..................... 24

4.2 Transverse profile changes with transverse wakefields .... 26

4.3 Emittance observations ............................... 29

4.4 Emittance minimization ................................. 31

4.5 Emittance collimation .................................... 33

4.6 Multibunch transverse effects ........................... 35

5. Acknowledgments ....................................... 37

6. References ................................................ 37

* Work supported by Department of Energy contract DE-AC03-76SF00515

Invited talk at the 1990 Joint US-CERN School on Particle Accelerators: Fronticrs of Particle Beams, Intensity Limitations, Hilton Head Island, SC, November 7-14, 1990. 


\title{
OBSERVATION OF HIGH CURRENT EFFECTS IN HIGH ENERGY LINEAR ACCELERATORS
}

\author{
John T. Seeman \\ Stanford Linear Accelerator Center \\ Stanford University \\ Stanford, California 94309
}

\section{Introduction}

The acceleration and transport of electron beams with high currents and low emittances are subjects studied very carefully for many operating accelerators and most future accelerators. For example, several Free Electron Lasers (FEL) [1] and the Stanford Linear Collider (SLC) [2] presently operate with high energy intense beams. The next generation of synchrotron light sources [3] and future linear colliders $[4,5,6,7]$ require a much higher degree of component precision and beam control. In this note the basic concepts governing the effects of high currents in accelerators are explored, including observations of high currents in present accelerators.

The effects of longitudinal wakefields on acceleration and energy spreads are studied first. Then, the transverse equations of motion are developed including the technique of BNS damping to control wakefield growth. These wakefield effects are investigated to characterize their influence on the control and observation of beam oscillations. Finally, the reduction of transverse wakefield effects is important for maintaining the emittances of intense beams.

\section{Longitudinal Effects}

\subsection{Acceleration}

The energy $E$ of particles accelerated in a linac after $n$ klystrons is given by [8]

$$
E(z)=E_{i n j}+\sum_{i=1}^{n}\left[\Delta E_{i} \cos \left(\phi_{i}+\phi(z)\right)+\Delta s_{i} \int_{z}^{\infty} W_{l}\left(z^{\prime}-z\right) \rho\left(z^{\prime}\right) d z^{\prime}\right]
$$


where $E_{i n j}$ is the injection energy, $\phi_{i}$ are the klystron phases-which are free parameters-, $\phi(z)=2 \pi z / \lambda_{R F}$, where $\lambda_{R F}$ is the $R F$ wavelength, $z$ is the longitudinal position along the bunch, $\Delta E_{i}$ is the maximum energy gain in the distance $\Delta \mathrm{s}_{\mathrm{i}}$ (one klystron), $\rho(z)$ is the longitudinal density distribution, and the last term on the right-hand side is the longitudinal wakefield contribution to the particle energy. The longitudinal wakefield contribution $W_{1}$ arises from all particles in the bunch preceding the one of interest. The longitudinal wakefield depends on the construction of the RF cavities and surrounding vacuum hardware. The longitudinal wakefield for the SLAC structure is shown in Figure 1 [9]. Typical accelerator parameters are $\Delta \mathrm{E}_{\mathrm{i}}=20 \mathrm{MeV} / \mathrm{m}, \lambda_{\mathrm{RF}}=10 \mathrm{~cm}$, and $\Delta \mathrm{s}_{\mathrm{i}}=12 \mathrm{~m} . \rho(\mathrm{z})$ is often a gaussian with sigma $\sigma_{\mathrm{z}}$.

\subsection{Energy spread}

The mean energy of the bunch $\langle\mathrm{E}>$ is an integral over the energy distribution.

$$
<E>=\int_{-\infty}^{\infty} E(z) \rho(z) d z
$$

The root-mean-square (rms) energy spread can also be calculated.

$$
\sigma_{E} / E=\frac{1}{\langle E\rangle}\left[\int_{-\infty}^{\infty}\left[E(z)-\langle E>]^{2} \rho(z) d z\right]^{1 / 2}\right.
$$

The energy spread for a low current beam is minimized when the bunch is centered on the crest of the RF wave form. An example of the resulting spectrum is shown in Figure 2 for the LIL linac at CERN [10]. The core of the beam has the highest energy and both the longitudinal head and tail particles have lower energies making a long low energy tail.

At high currents the longitudinal wakefield decelerates the longitudinal tail of the bunch (generally). Thus, in order to minimize the energy spread the mean phase of the bunch is moved forward of the RF crest. This process can be seen schematically in Figure 3. The energy distribution in the bunch develops a complicated shape due to the nonlinear wakefield, particle density distribution, and the RF sine wave. These distributions have been calculated for the SLC linac for low and high currents and are shown in Figure 4. The fact that the minimum spectrum is obtained at a phase forward of the RF crest is easy to verify in a linac. An example for the SLC is shown in Figure 5 [11]. The "double horned" energy distributions indicated in Figure 3 for high currents have also been seen in the SLC (Figure 6) at about $3 \mathrm{x}$ $10^{10} \mathrm{e}^{-}$with $\sigma_{\mathrm{z}}=1 \mathrm{~mm}$. The changes in the optimum linac phase for the minimum energy spread as a function of current are shown in Figure 7. The required phase shift can be on the order of 10 to 15 degrees. This shift allows phase jitter in the klystrons to produce energy jitter in a linac. 


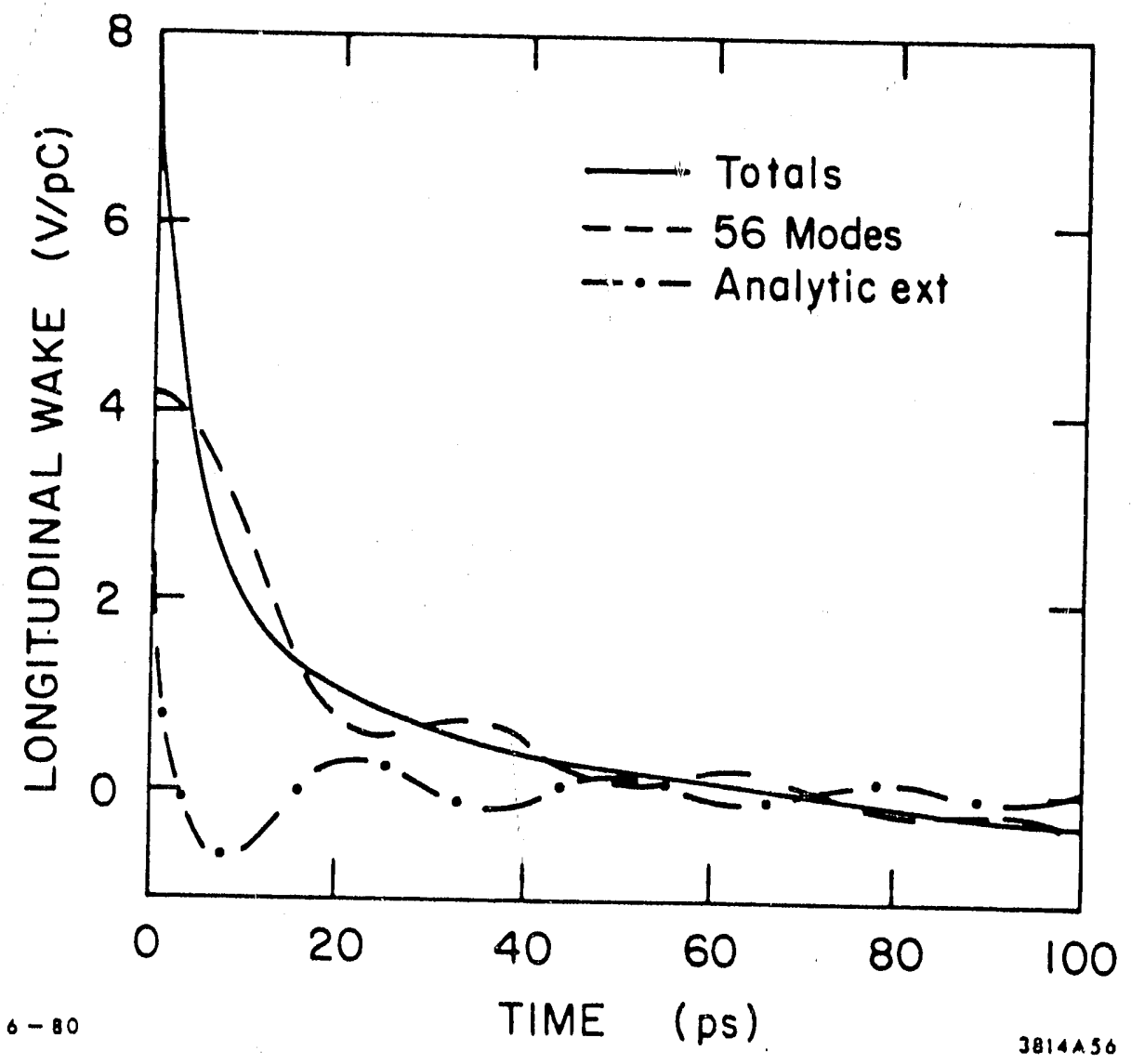

Figure 1 Longitudinal wake potential of the SLAC RF structure for a single cell as experienced by a test particle following a single electron.

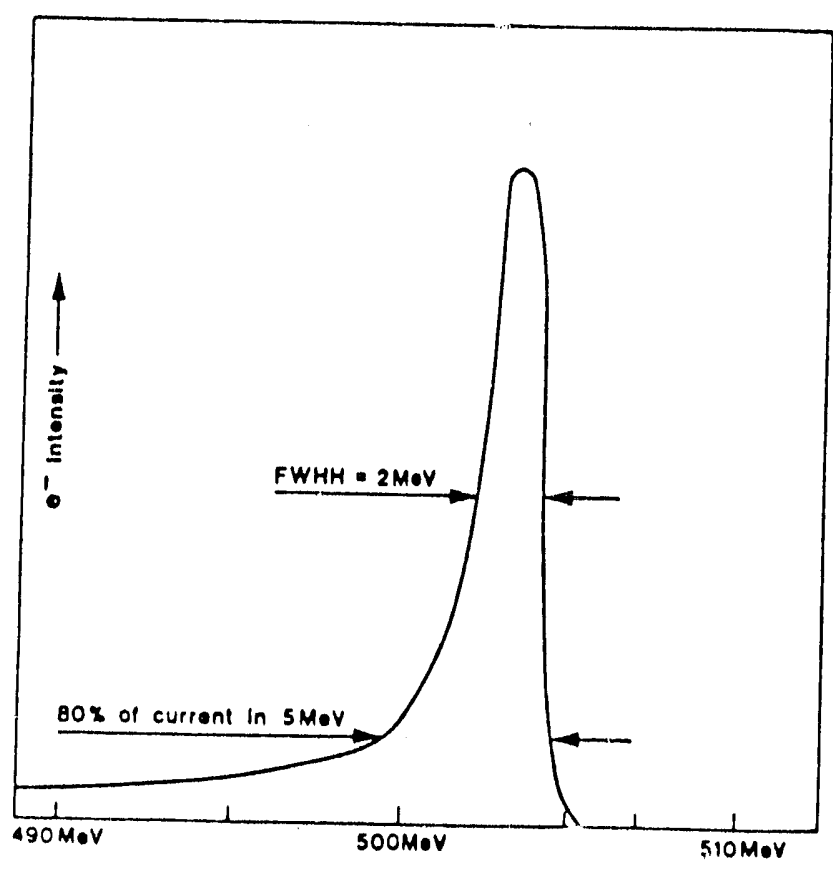

Figure 2 Measured energy spectrum for a low charge bunch in the LIL linac at CERN. 

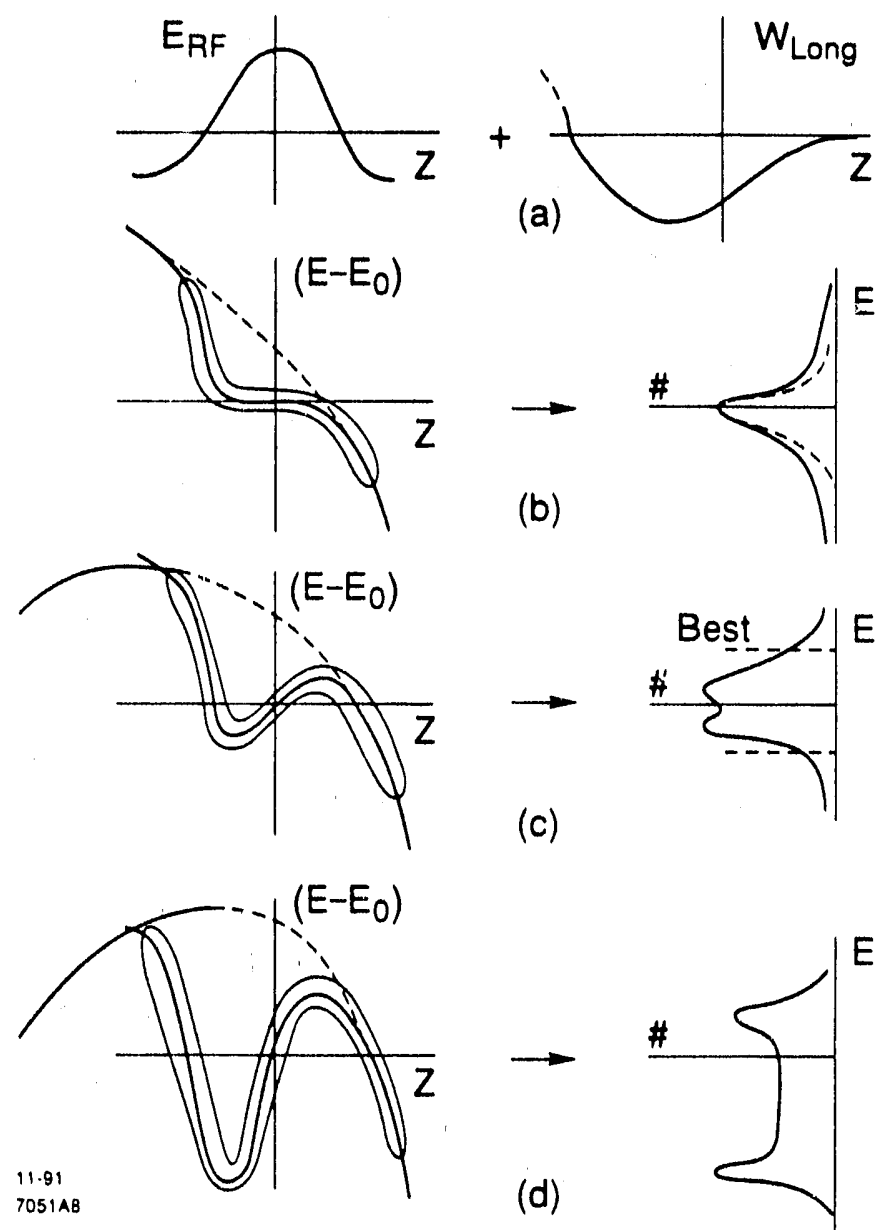

(c)

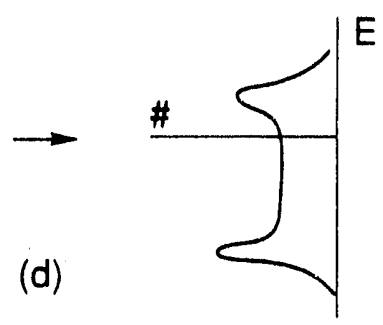

Figure 3 Schematic view of the effect of the overall linac RF phase on the energy spectrum of bunches with high charges including longitudinal wakefields (a). The energy versus longitudinal position displays on the left $(b, c, d)$ lead to the energy density distributions on the right. The optimum phase places as many particles as possible near the mean energy. The optimum produces a double peaked distribution (c). With longitudinal wakefields the optimum phase of the bunch is noticeably ahead of the RF crest.

Occasionally, the longitudinal bunch distributions are not true gaussians and careful integrals are required to extracted the energy spreads [12]. In practice near gaussians and gaussians are difficult to distinguish experimentally.

\subsection{Multibunch longitudinal effects}

When a linac accelerates multiple bunches, the longitudinal loading affects the energy differences between the bunches. Trailing bunches see a lower accelerating field. The energy extracted from the RF cavities by the beam must be replenished by an RF power source but there is a finite filling time. The methods to equalize the bunch energies are to stagger time the RF pulses to add extra energy between bunches or to change the klystron pulse amplitude with time. The former is being considered for the next 


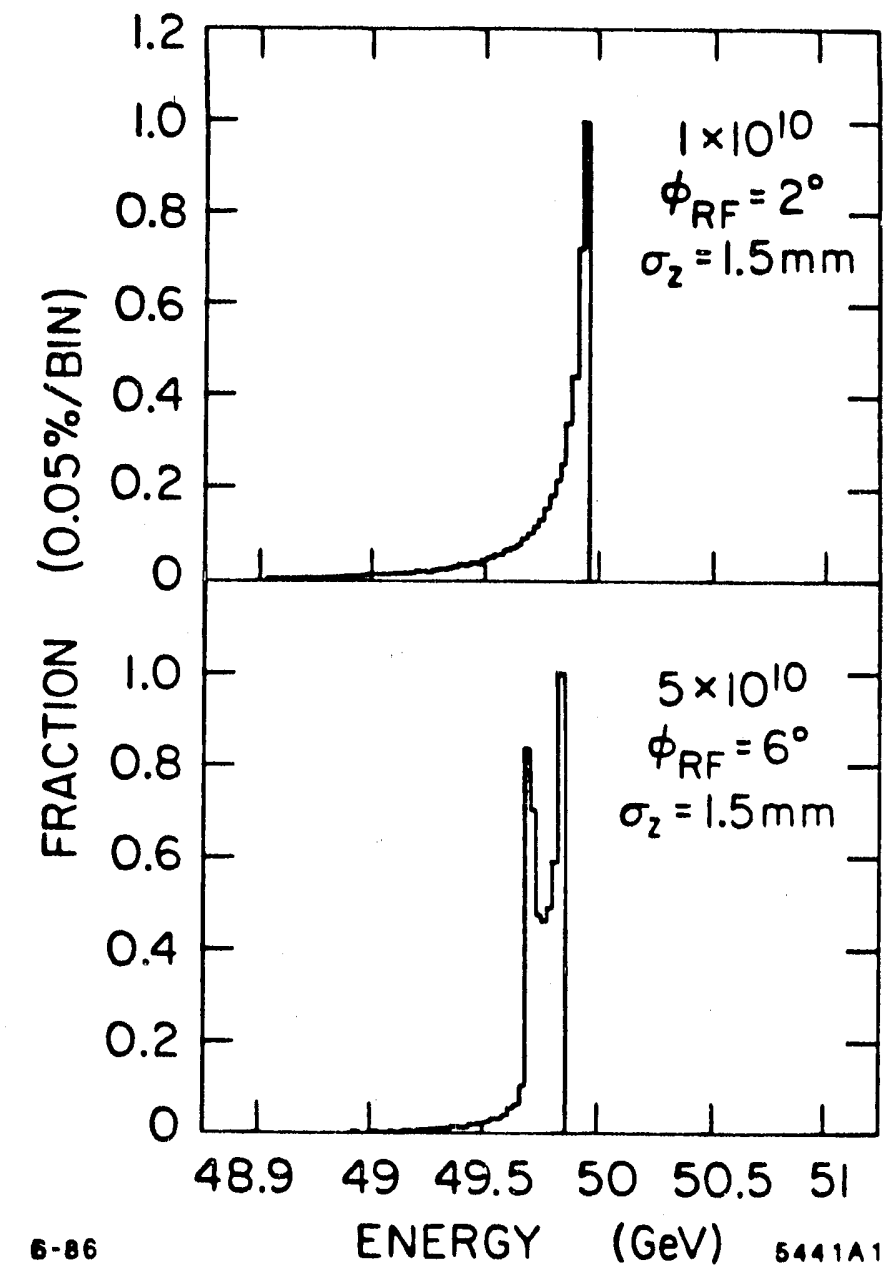

Figure 4 Simulated energy spectra for the SLC linac showing optimized cases for low charge (upper) and high charge (lower). The high charge spectrum has a complicated shape affecting the transport of low emittance beams.

linear collider [13] and the latter is used for making the two colliding bunches in the SLC have equal energies [11]. An example of multibunch longitudinal loading without compensation is shown in Figure 8 where each trailing bunch has lower and lower energy. The center bunches in the beam have higher charge than either end and the corresponding energy spacings are larger. The method to compensate the two colliding bunches in the SLC is shown in Figure 9, where the pulsed SLED system [14] provides a time varying field.

\section{Transverse Oscillations}

\subsection{Equation of motion with transverse wakefields}

The transverse equation of motion for particles under the influence of transverse wakefields is

$$
\frac{d^{2}}{d s^{2}} x(z, s)+k^{2}(z, s) x(z, s)=\frac{r_{e}}{\gamma(z, s)} \int_{z}^{\infty} d z^{\prime} \rho\left(z^{\prime}\right) W_{r}\left(z^{\prime}-z\right) x\left(z^{\prime}, s\right),
$$




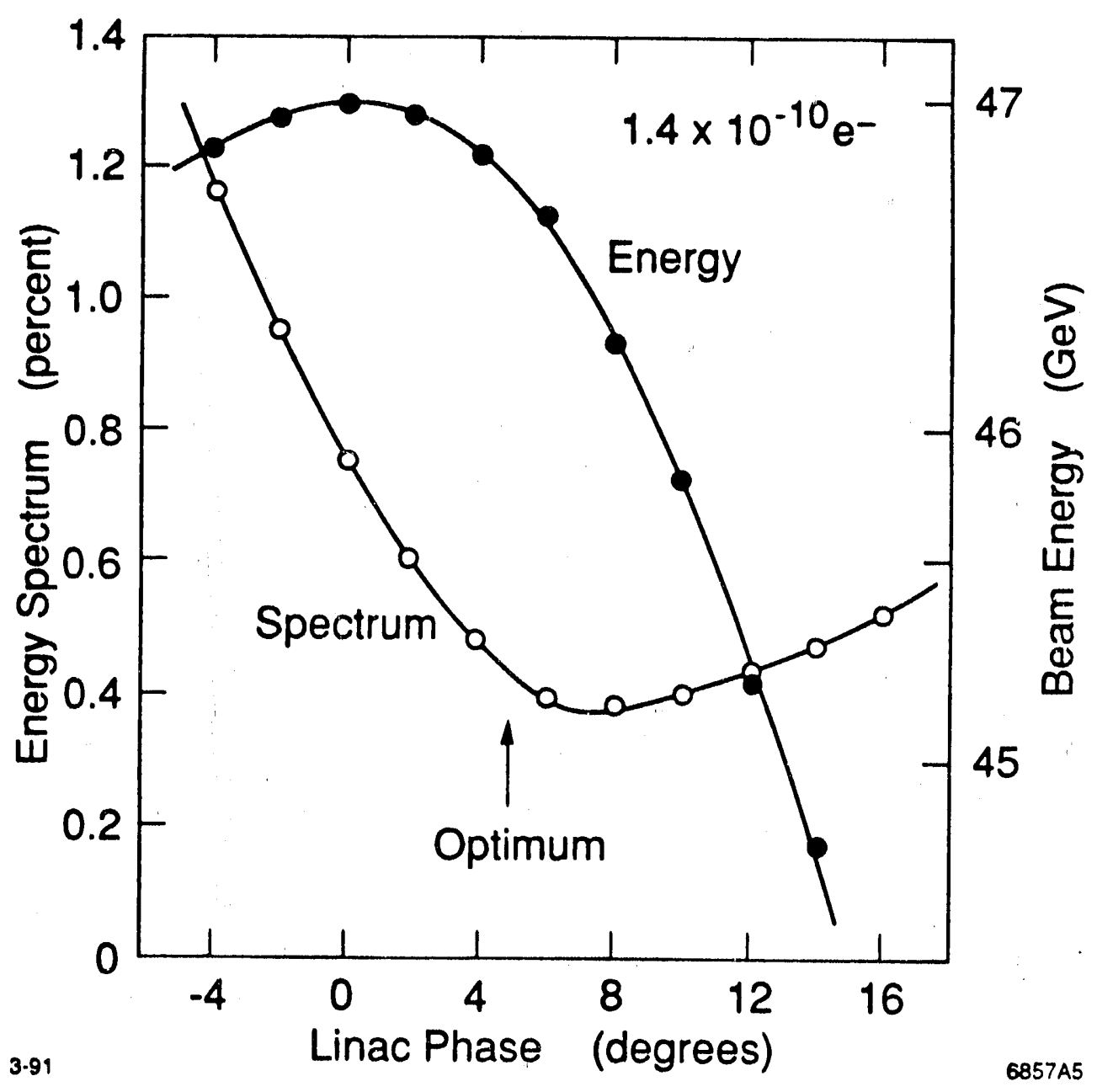

Figure 5 Measured energy and energy spectrum of a bunch in the SLC linac with medium charge as a function of RF phase. The peak energy and smallest rms energy spread are separated by several degrees as expected from longitudinal wakes.

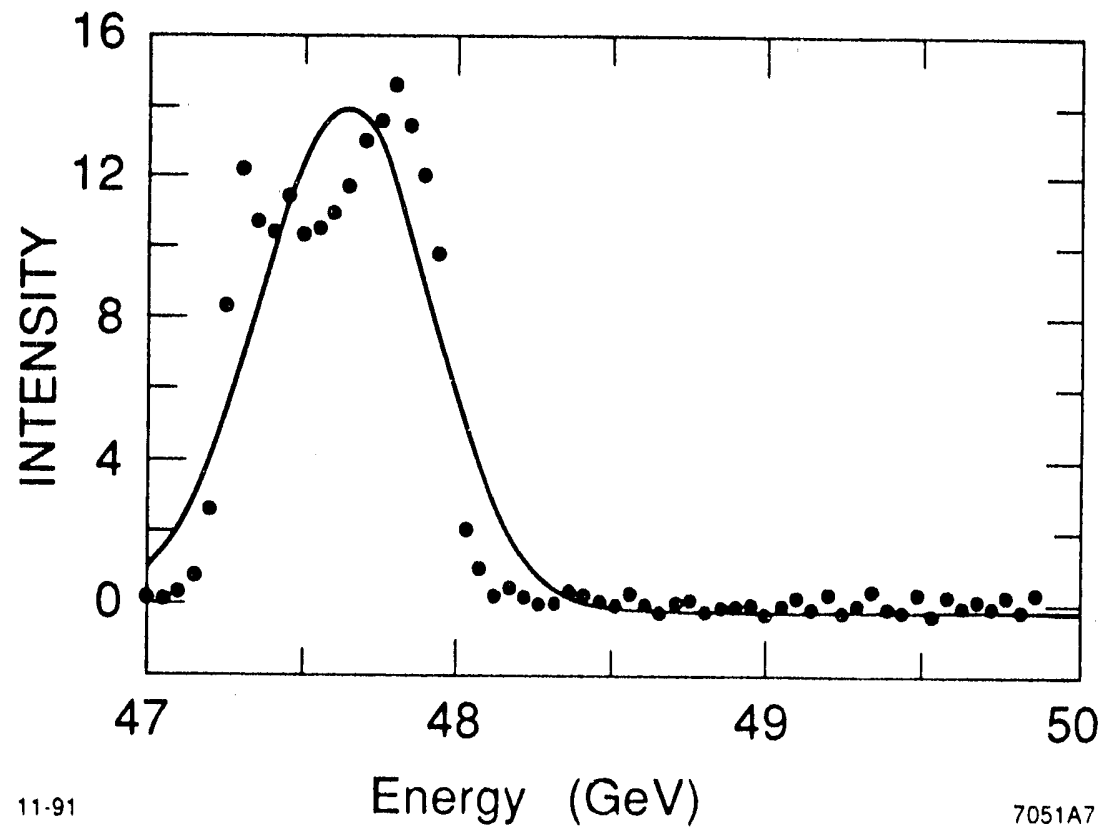

Figure 6 At high charge the measured energy spectrum in the SLC has the characteristic double peaked structure as schematically illustrated in Figure 3. 


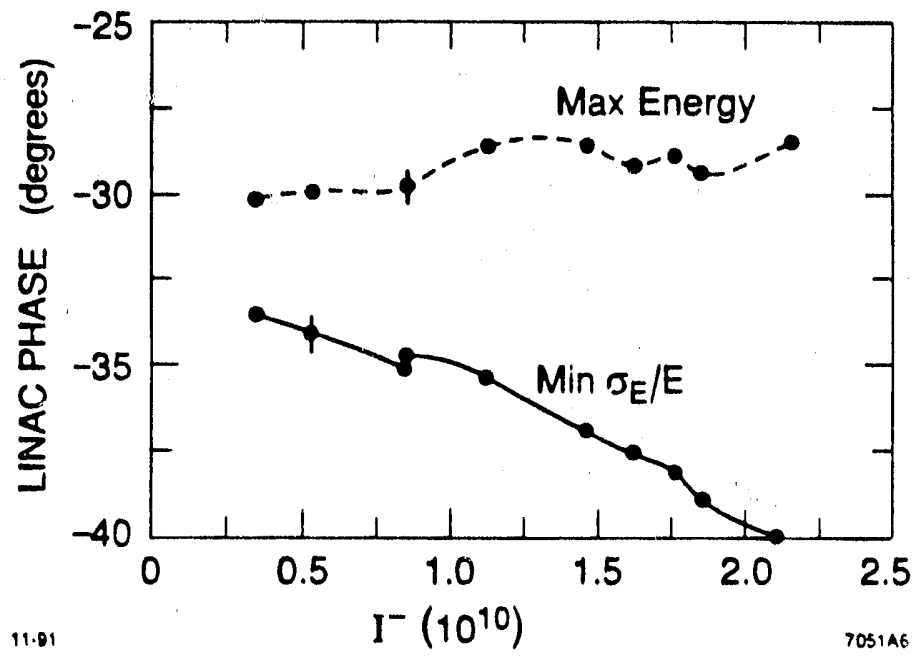

Figure 7 Measured RF phases for the maximum energy of the core of the beam as well as the minimum energy spread of the whole bunch as a function of charge. The phase of the peak energy remains nearly the same but the phase for the minimum energy spread shifts considerably forward at high intensity.

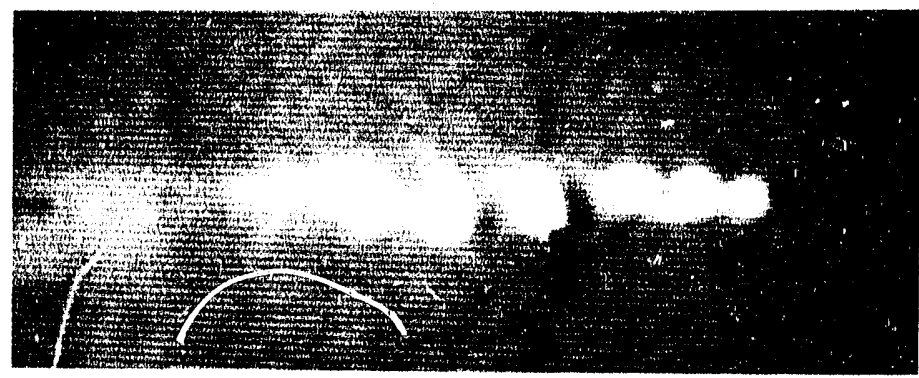

Figure 8 Photographed position of a train of six electron bunches in the SLAC linac at $8 \mathrm{GeV}$ on a profile monitor in a dispersive region $(\eta=1 \mathrm{~m})$ after $800 \mathrm{~m}$ of $R F$ accelerator. The bunches fill six adjacent $R F$ buckets. The position offsets indicate the long range longitudinal loading of each bunch on all trailing bunches. Each bunch charge is about $0.5-1.0 \times 10^{9} \mathrm{e}^{-}$. The horizontal full scale is $2.5 \mathrm{~cm}$. The dark mark is an artifact of the measurement.

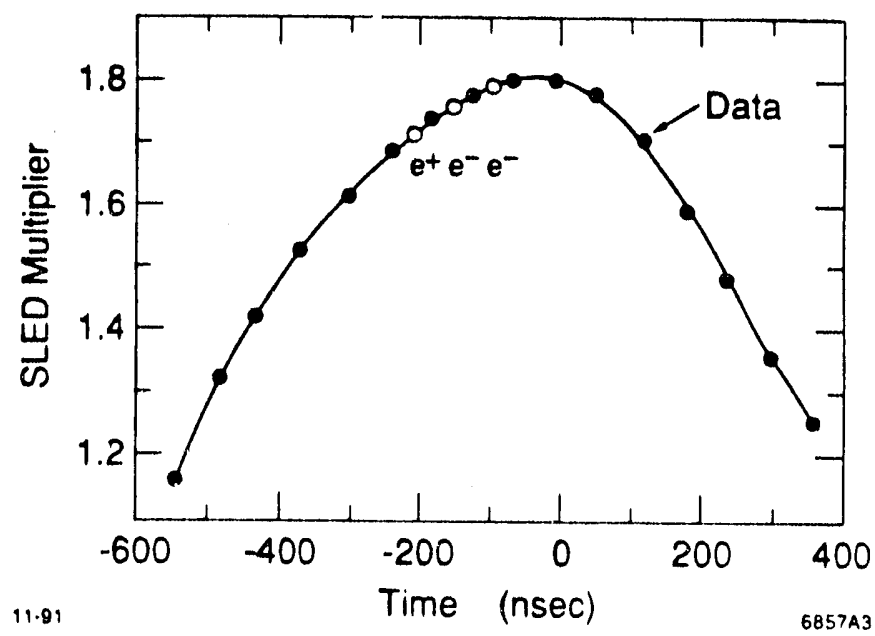

Figure 9 Measured SLED RF wave form indicating the timing of the three SLC bunches. The global timing of the three bunches is adjusted to alleviate any encrgy changes from charge differences and longitudinal loading between the bunches. 
where $\mathrm{x}$ is the transverse particle displacement which depends on where in the bunch the particle is located and where along the accelerator the bunch is located, $k$ is the lattice strength, $\rho$ is the longitudinal particle density, $\gamma=E / \mathrm{mc}^{2}, \mathrm{r}_{\mathrm{e}}$ is the classical electron radius, $\mathrm{s}$ is the distance long the accelerator, and $\mathrm{W}_{\mathrm{r}}$ is the transverse wakefield which depends on the separation of the leading and trailing particles. $\mathrm{W}_{\mathrm{r}}$ grows nearly linearly for most bunches with millimeter bunch lengths as can be seen in Figure 10 calculated for the SLAC structure [9]. The acceleration is generally linear, $\gamma(s)=\gamma(0)+G s$, where $G$ is the accelerating gradient. In Equation (4) the distance to double the energy is assumed to be long compared to the betatron wavelength, or $\mathrm{k}>\mathrm{G}$.

The driving force on the right hand side is proportional to the transverse offset of all the leading particles in the RF structure of the accelerator. Thus, there is the potential for resonant excitation of the back of the bunch by the head. The wakefield excitation of the bunch through an oscillation can be seen schematically in Figure 11. There are many mechanisms to start an oscillation and the resulting resonant excitation. The energy spread in the bunch affects the equation of motion. Also, the motion will develop differently if the initial deflection is made at different locations in the accelerator.

If the transverse wakefields are set to zero, then the solution to Equation (4) is the standard betatron oscillation. The expected beam oscillation given a dipole deflection $\Delta \theta$ at location ' 0 ' with acceleration is

$$
x(s)=\Delta \theta \beta(s) \sqrt{E(0) \beta(0) / E(s) \beta(s)} \sin (\psi(s)-\psi(0))
$$

where $\beta$ is the beta function, and $\psi(s)$ is the betatron phase to position s. Note that the betatron oscillation damps with the square root of the energy.

The equation of motion involves only one geometrical plane and assumes no horizontal-vertical coupling. This is often a reasonable assumption for round beam accelerators (see Figure 12) and is a strong requirement for a future collider [7].

An instructive exercise is to solve Equation (4) for a beam with three longitudinal slices without acceleration, i.e. a three particle model. If the bunch has a total charge of $N$, then the center ' $c$ ' slice is assigned N/2 charges and the head ' $h$ ' and tail ' $t$ ' slices have $N / 4$ charges each. They are all spaced apart by $\sigma_{z}$. The three equations of motion assuming a linear wakefield become

$$
\begin{aligned}
& x_{h}+k_{\beta}^{2} x_{h}=0 \\
& x_{c}+k_{\beta}^{2} x_{c}=B x_{h} \\
& x_{t}+k_{\beta}^{2} x_{t}=2 B x_{h}+2 B x_{c}
\end{aligned}
$$

where $\mathrm{B}$ is proportional to the bunch charge and the transverse wakefield.

$$
\mathrm{B}=-\mathrm{e} \frac{\mathrm{N} \mathrm{W}_{\mathrm{r}} \sigma_{2}}{4 \mathrm{E}_{0}}
$$




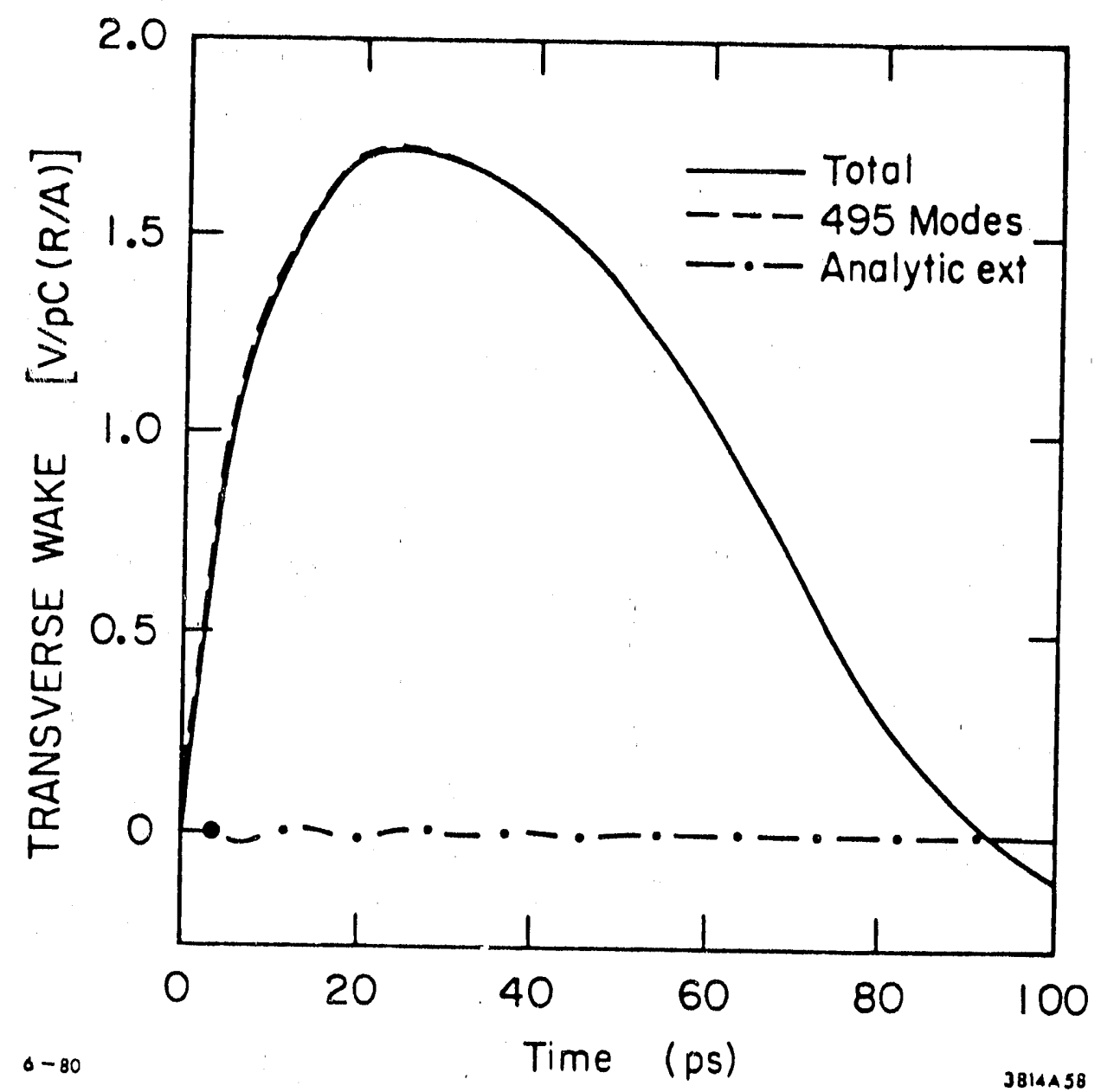

Figure 10 Transverse wake potential produced by a single electron in one RF cell of the SLAC linac.

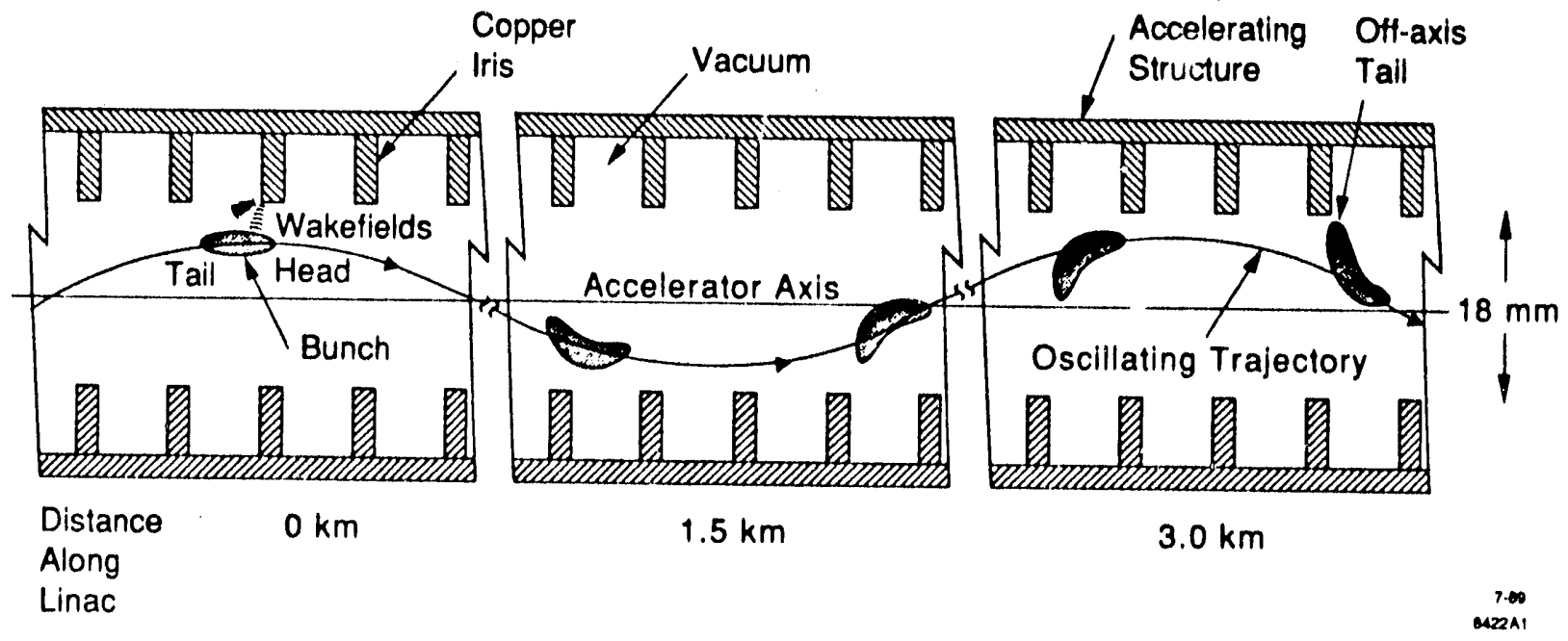

Figure 11 Schematic illustration showing the growth in oscillation amplitude of the longitudinal tail of a beam driven by transverse wakefields from the leading portions of the bunch. Trajectory correction downstream has difficulty converging as some part of the beam is always off axis driving trailing particles to larger amplitudes. 
The solutions to the above equations assuming an initial deflection at $s=0$ of $\theta_{0}=k_{\beta} x_{0}$ are

$$
\begin{aligned}
& \frac{x_{h}}{x_{0}}=\sin \left(k_{\beta} s\right) \\
& \frac{x_{c}}{x_{0}}=\left(1+\frac{B}{4 k_{\beta}^{2}}\right) \sin \left(k_{\beta} s\right)-\frac{B}{2 k_{\beta}} s \cos \left(k_{\beta} s\right) \\
& \frac{x_{t}}{x_{0}}=\left[1+\frac{2 B}{k_{\beta}^{2}}\right] \sin \left(k_{\beta} s\right)-\frac{2 B}{k_{\beta}} s \cos \left(k_{\beta} s\right)-\frac{B^{2}}{4 k_{\beta}^{2}} s^{2} \sin \left(k_{\beta} s\right)
\end{aligned}
$$

There are three important conclusions: 1) The variable $\mathrm{x}_{0}$ factors from all the solutions, which means that the solutions are linear in the amplitude. 2) Each additional slice adds one higher power of $B\left(\right.$ or $W_{r} N$ ) and $\mathrm{s}$, indicating the nonlinearity in bunch charge and distance along the accelerator. 3) The core lags 90 degrees behind the head. The tail, ultimately, lags 180 degrees behind the head. Conclusion 2 suggests an exponential growth of the tail of the beam in the limit of many slices.

This resonant blowup at high currents was observed in the SLC as is shown in Figure 13. Simulations and observations show good agreement indicating an approximate exponential trajectory increase with distance [15]. In this condition the SLC was very susceptible to injection jitter.

\subsection{Transverse wakefield (BNS) damping}

A technique to control this resonant growth is called BNS damping (named after the inventors: V. Balakin, A. Novokhatsky, and V. Smirnov)[16]. BNS damping lowers the energy of the back of the bunch by accelerating the bunch behind the crest of the RF early in the linac and then ahead of the RF crest downstream to restore the energy spread at the end. With the back of the bunch low in energy, the wakefields forces in Equation (4), which act to defocus particles, are counterbalanced by the increased quadrupole focusing of the back of the bunch because of its low energy. The resonant excitation is then reduced. It is not possible to perfectly cancel the forces over the whole bunch so some enlargement is expected.

Again, it is instructive to find the solutions to the three particle model but with BNS damping added. BNS damping enters the equations in the lattice constants $k_{\beta}$. The energies of the three particles are

differert and so too are their lattice constants. $k_{\beta}$ for the head is set to $k_{\beta}+\alpha$, for the core $k_{\beta}$, and for the tail $k_{\beta}-\delta$. The solutions to the three equations with $x_{0}=\theta_{0} /\left(k_{\beta}+\alpha\right)$ are

$$
\begin{aligned}
& \frac{\mathrm{x}_{\mathrm{h}}}{\mathrm{x}_{0}}=\sin \left(\left(\mathrm{k}_{\beta}+\alpha\right) \mathrm{s}\right) \\
& \frac{\mathrm{x}_{\mathrm{c}}}{\mathrm{x}_{0}}=\sin \left(\mathrm{k}_{\beta} \mathrm{s}\right)+D\left[\left(\mathrm{k}_{\beta}+\alpha\right) \sin \left(\mathrm{k}_{\beta} \mathrm{s}\right)-\mathrm{k}_{\beta} \sin \left(\left(\mathrm{k}_{\beta}+\alpha\right) s\right)\right]
\end{aligned}
$$

where

$$
D=\frac{B}{k_{\beta}\left(\left(k_{\beta}+\alpha\right)^{2}-k_{\beta}^{2}\right)}
$$



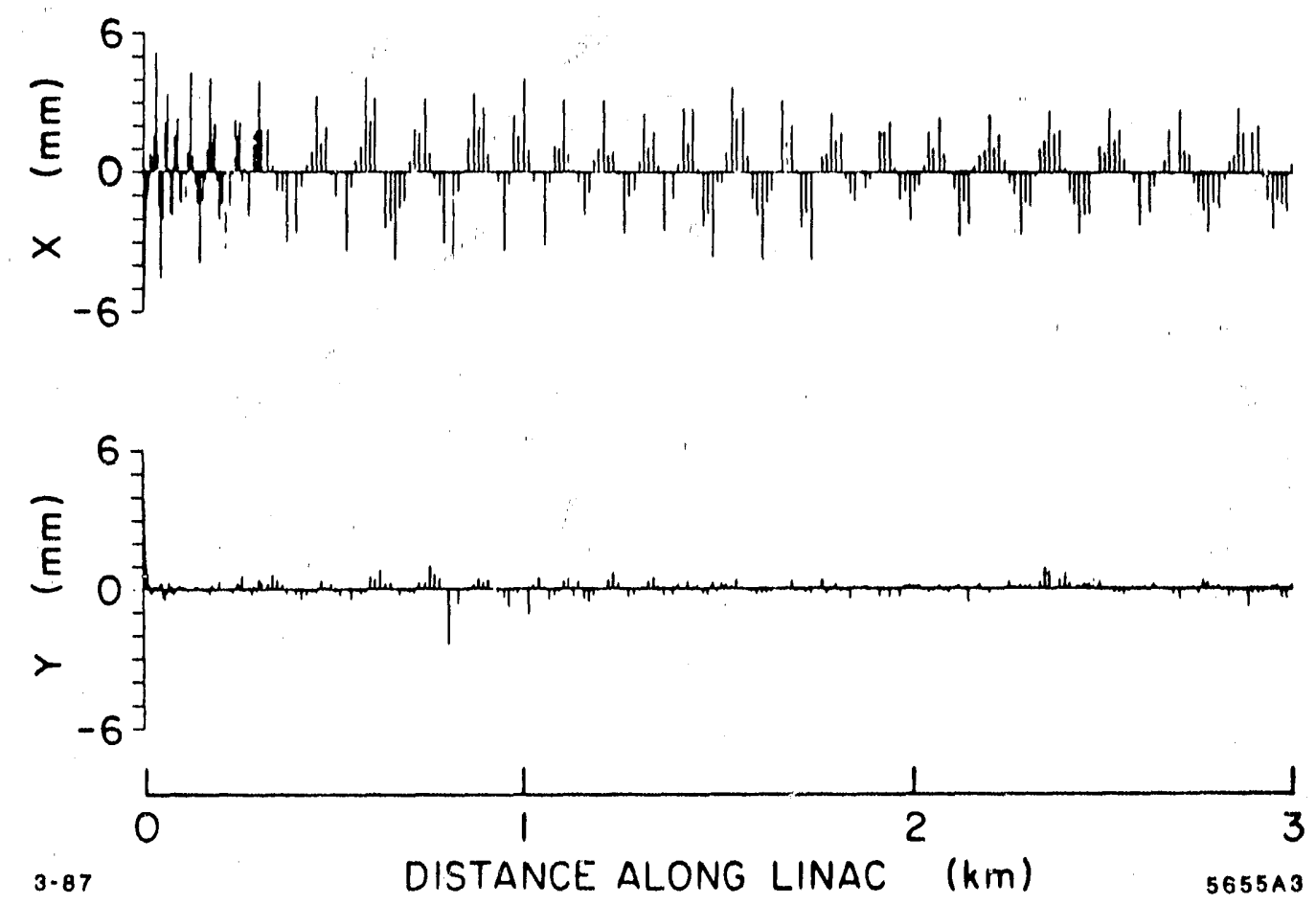

Figure 12 A measured horizontal betatron oscillation in the linac induced by a dipole magnet early in the linac. Note that the oscillation remains coherent and does not couple into the vertical plane showing the independence of the transport equations $\left(6 \times 10^{9}\right.$ electrons at $\left.42 \mathrm{GeV}\right)$.
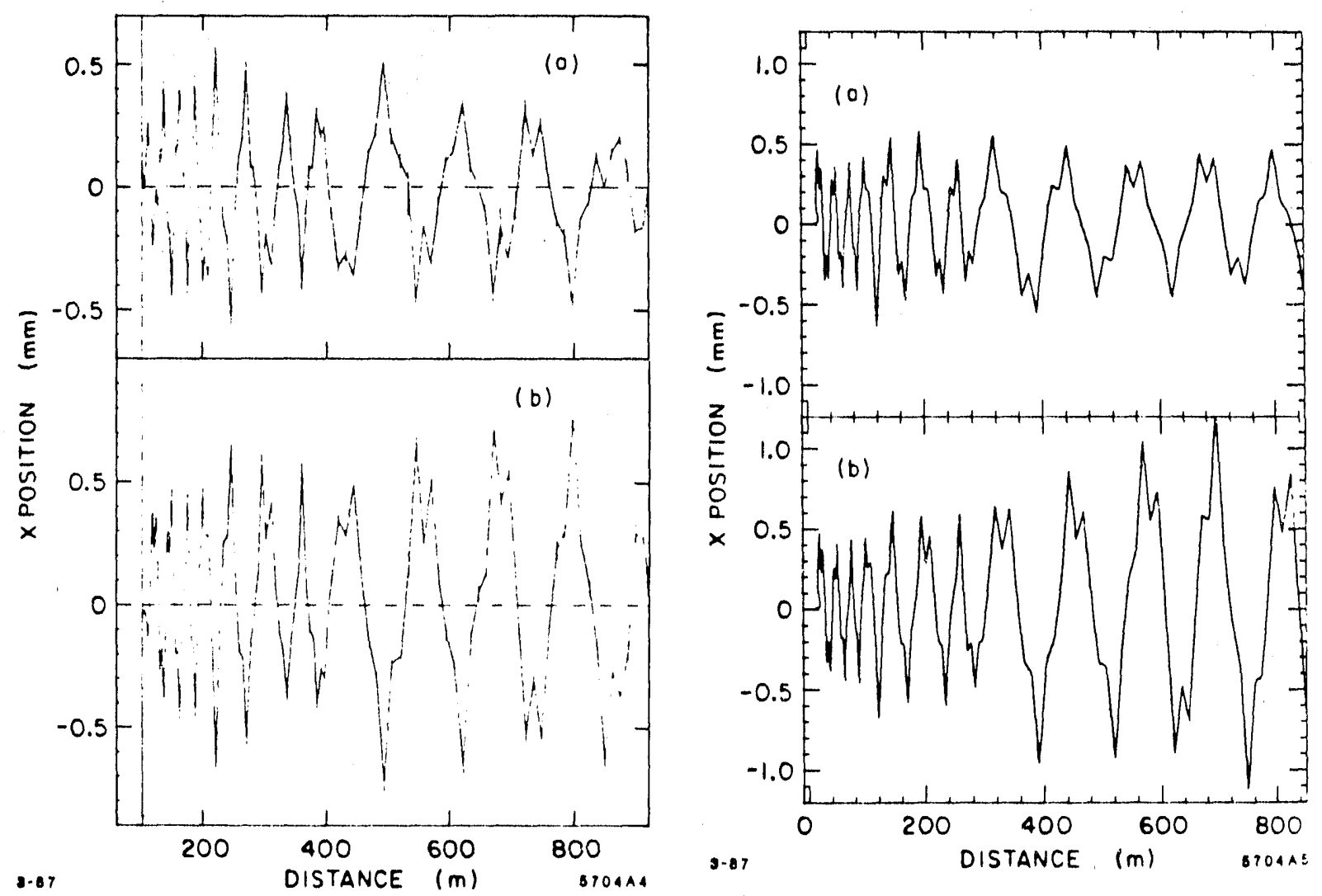

Figure 13 Left: Measured trajectories of an accelerated e'ectron beam due to a 3 gauss-m dipole deflection. Right: Simulated trajectories of the electron beams on the left. The bunch lengths are $1 \mathrm{~mm}$. The upper curves are for $8 \times 10^{9} \mathrm{e}^{-}$; the lower curves are for $2.2 \times 10^{10}$ $\mathrm{e}-$ No BNS damping phases are used. Note that the simulation and measurements agree and that at $2.2 \times 10^{10} \mathrm{e}^{-}$the trajectory is starting to grow exponentially with distance. 


$$
\begin{array}{r}
\frac{x_{1}}{x_{0}}=\sin \left(\left(k_{\beta}-\delta\right) s\right)+G\left[\left(k_{\beta}+\alpha\right) \sin \left(\left(k_{\beta}-\delta\right) s\right)-\left(k_{\beta}-\delta\right) \sin \left(\left(k_{\beta}+\alpha\right) s\right)\right] \\
+F\left[k_{\beta} \sin \left(\left(k_{\beta}-\delta\right) s\right)-\left(k_{\beta}-\delta\right) \sin \left(k_{\beta} s\right)\right]
\end{array}
$$

where

$$
\begin{aligned}
& G=\frac{2 B\left[1-B /\left[\left(k_{\beta}+\alpha\right)^{2}-k_{\beta}^{2}\right]\right.}{\left(k_{\beta}-\delta\right)\left[\left(k_{\beta}+\alpha\right)^{2}-\left(k_{\beta}-\delta\right)^{2}\right]} \\
& F=\frac{2 B(1+H)}{\left(k_{\beta}-\delta\right)\left[k_{\beta}^{2}-\left(k_{\beta}-\delta\right)^{2}\right]}
\end{aligned}
$$

and

$$
\mathrm{H}=\frac{B\left(\mathrm{k}_{\beta}+\alpha\right)}{\mathrm{k}_{\beta}\left[\left(\mathrm{k}_{\beta}+\alpha\right)^{2}-\mathrm{k}_{\beta}^{2}\right]} .
$$

After this tedious work, the conditions for BNS damping can be determined. To make the head and core particles oscillate tugether, $\alpha$ is adjusted to make identical trajectories.

$$
\alpha=\mathrm{eNW}_{\mathrm{r}} \sigma_{\mathrm{z}} /\left(4 \mathrm{E}_{0} \mathrm{k}_{\beta}\right) \quad \text { (BNS condition) }
$$

This is the standard BNS condition for a two particle model. The core wants to have an energy that makes the lattice appear stronger, i.e. a lower energy. To make the third particle oscillate like the first two then

$$
\delta=4 \alpha . \quad \text { (optimum) }
$$

This solution indicates that the encrgy differences over the bunch need careful tailoring. This is the essence of the notion of autophasing described in Section 3.4. Finally, RF focusing can be used to provide different lattice strengths to the various parts of the bunch instead of a head-tail energy difference. RF focusing may be used in a future collider [17].

The strong effect of this transverse damping is shown in Figure 14 where beams with and without BNS damping are made to oscillate in the SLC [18]. BNS damping works very well and is used continuously. With full BNS damping conditions, oscillations of a bunch have been studied in detail. Four measured oscillations, with identical initial conditions. are shown in Figure 15 for four different bunch currents. The BNS settings are for $2.5 \times 10^{10}$ particles, where the first 48 klystrons have a phase of -20 degrees and the remaining klystrons have a phase of +15 degrees. In all cases the overall linac phase was adjusted to make the energy spectrum correct at the end of the accelerator. At low current the oscillation decoheres because of the large energy spread. At $2.5 \times 10^{10}$ particles BNS damping is nearly correct because the oscillation damps approximately was the square root of the energy, as expected. At this setting the bunch resists wakefield emittance enlargement. At the highest current the BNS conditions are no longer effective and the oscillation starts to grow strongly at the end of the linac, similar to the examples in Figure 14. Thus, the BNS conditions must be matched to the beam current and lattice to make the technique most successful. However, if the klystron phases are significantly misphased from the desired values, bizarre oscillations can result. An example is shown in Figure 16 where strong beating is observed. 


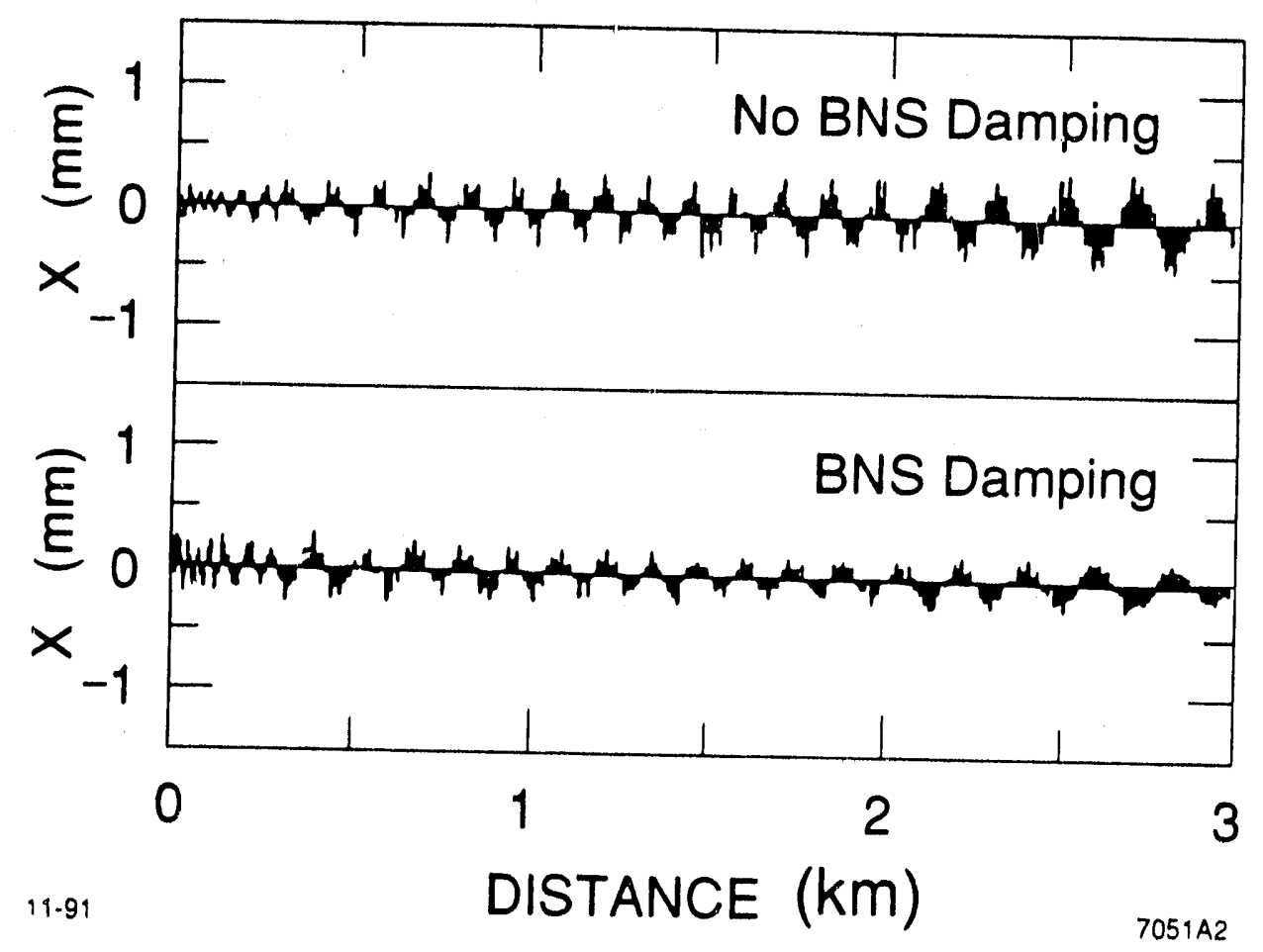

Figure 14 Measured oscillations in the SLC linac with and without transverse wakefield damping (BNS). The bunch charges are $2 \times 10^{10} \mathrm{e}^{-}$. The upper plot shows the exponential growth of the oscillation without BNS damping and the lower plot shows that with BNS damping. In this example, the improvement in the ratio from front to end of the linac is a factor of ten.

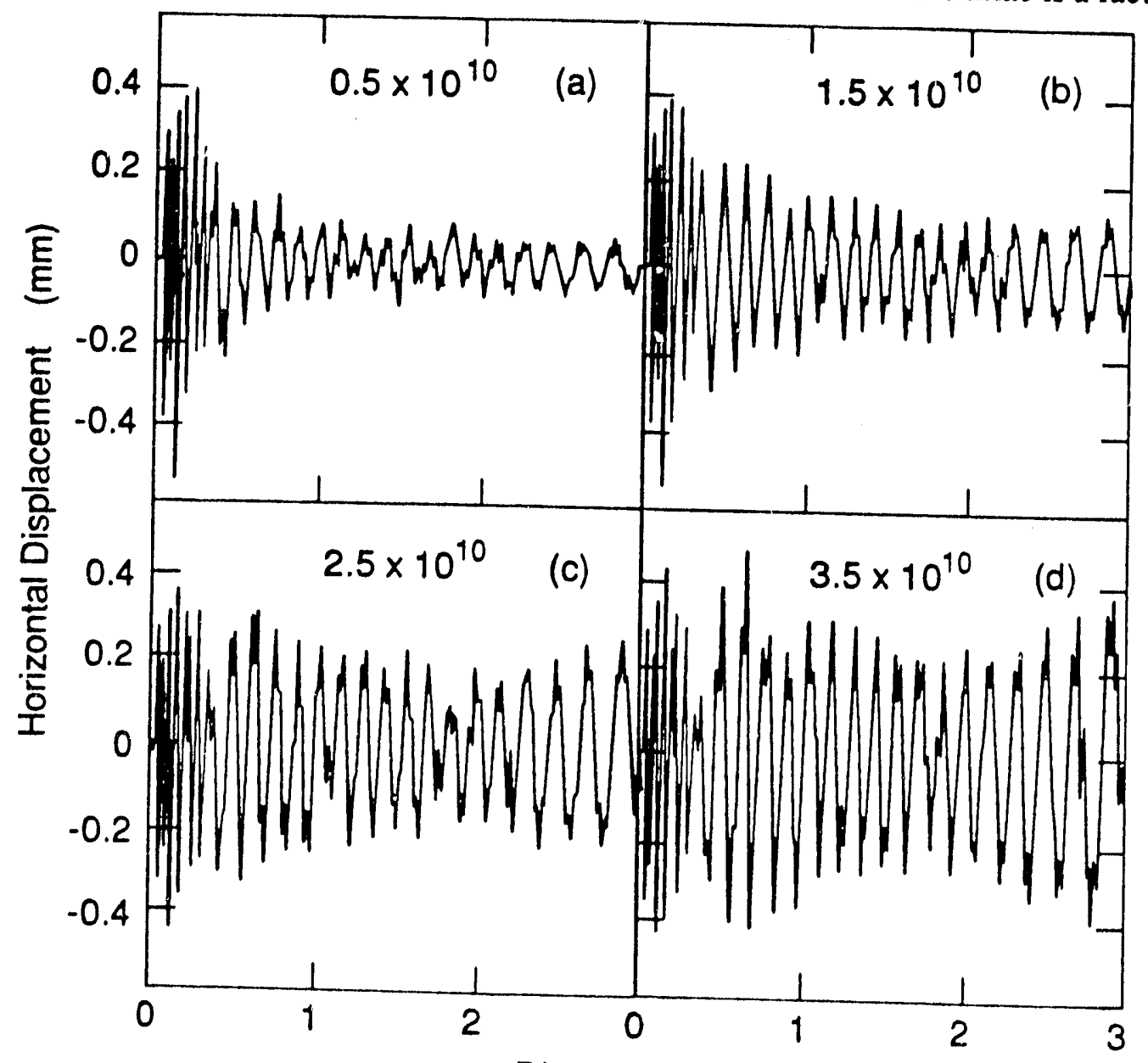

Figure 15 Observed single bunch oscillations along the SLC linac versus charge for identical dipole changes. Standard BNS conditions for $3 \times 10^{10} \mathrm{e}^{-}$are used in all cases with the overall linac phase adjusted to make a small energy spectrum at $47 \mathrm{GeV}$. Note that at high charge, damping from acceleration is not as strong as wakeficld growth in the latter half of the linac. 


\subsection{Analysis of oscillations}

A beam oscillation can be used to determine lattice and beam parameters. An example of an oscillation with is fit using a global energy factor as a parameter is shown in Figure 17 [19]. This type of analysis can aid in determining if klystrons need phasing, if quadrupoles are misadjusted, or if transverse wakefield effects are proper.

By recording a beam trajectory and fitting the oscillation using Equation (4), an effective lattice strength can be determined. A plot of the change in the effective lattice strength with current for the SLC is displayed in Figure 18. In an accelerator with 30 betatron wavelengths, a strength change of a few percent is quite noticeable. Conversely, Equation (4) can be used to determine an effective beam current, assuming a longitudinal density profile. This study was done and the currents from the fits versus the actual beam currents for a particular SLC accelerator condition are plotted in Figure 19. Although the data and the predictions do not fully agree, there is no doubt about the strong effect on the apparent betatron frequency [19].

\subsection{Autophasing}

The cancellation of wakefield forces by BNS damping may be exploited further, if a concept called autophasing can be made practical [20]. By careful arrangement of the bunch charge density based on knowledge of the local beam energy, lattice, bunch length, and RF structure, nearly all particles in the bunch can be made to follow exactly the same trajectory. The conditions for this behavior can be derived by substituting an identical oscillation $\left(x_{=}=x_{0} \sin (k s)\right)$ into Equation (4) for all particles, and cancelling position terms on both sides [8]. The resulting autophasing condition is

$$
k^{2}(z, s)=k_{0}^{2}+\frac{r_{0}}{\gamma(z, s)} \int_{z}^{\infty} d z^{\prime} \rho\left(z^{\prime}\right) W_{r}\left(z^{\prime}-z\right)
$$

where $k_{0}$ is the nominal lattice strength and $k(z, s)$ would be the lattice strength for all particles if transverse wakefield forces were removed. With wakefields and the above condition, all particles have the same effective betatron frequency. The difficulty is that this condition must hold for all particles in the bunch, even the ones far forward off the RF crest, and at all locations along the accelerator. The adjustable parameters available are the bunch current, the lattice strength, the klystron energy profile, the klystron phases, and the longitudinal density profile. However, of all these variables, the longitudinal density profile is the most useful.

Using the two particle model in Equation 10, one can calculate the reduction in energy needed by the second particle to achieve autophasing. This calculation was done for the SLC and is shown in Figure 20 [19]. The early part of the SLC has several lattice discontinuities which require beam energy spread changes which are more rapid than can be practically made. 

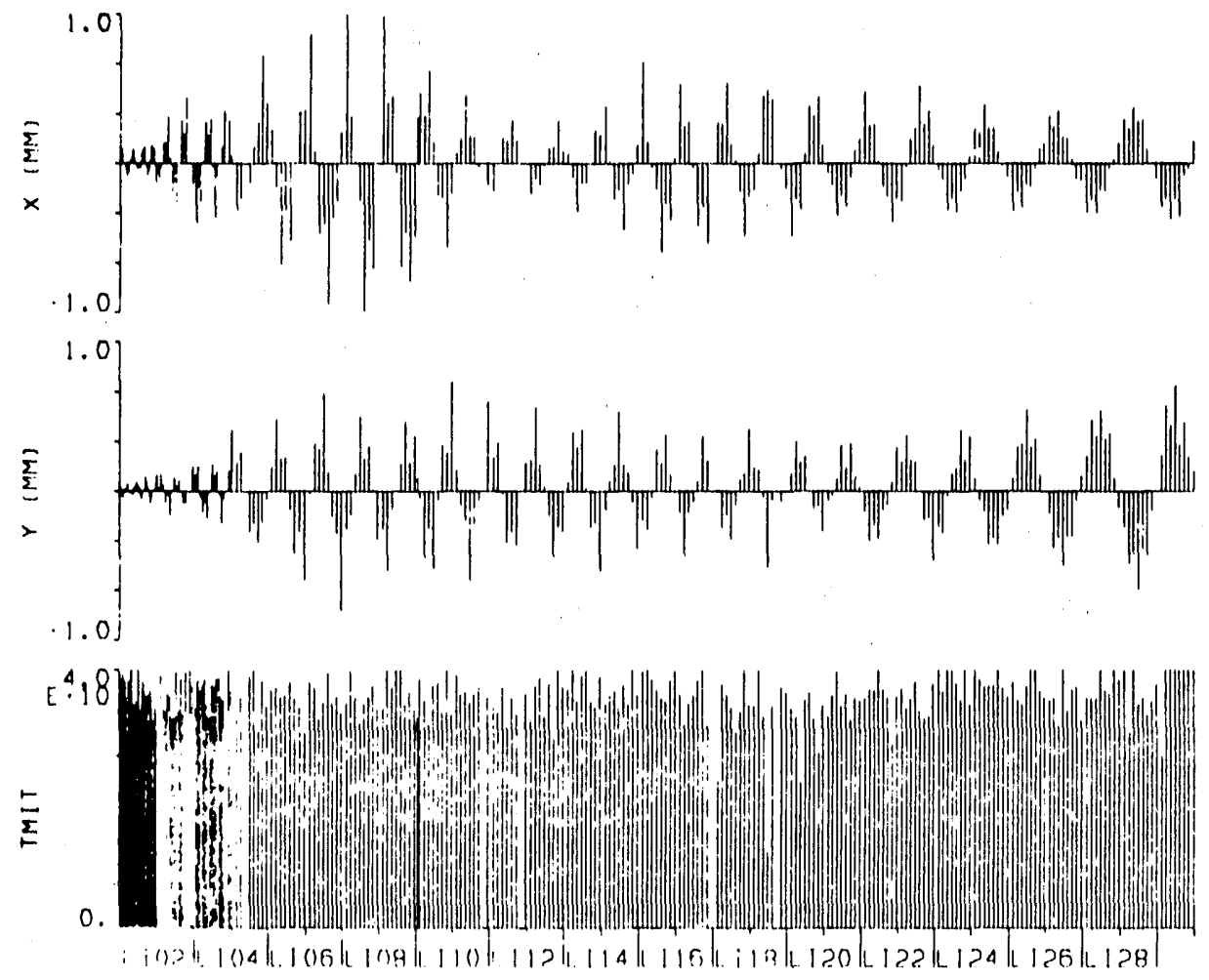

Figure 16 Observed dipule driven oscillations along the SLC linac of a beam with an abnormally large energy spectrum. The beating of the observed positions arises from the head and tail positions being on the same or opposite side of the axis. Large chromatic emittance enlargement also results.

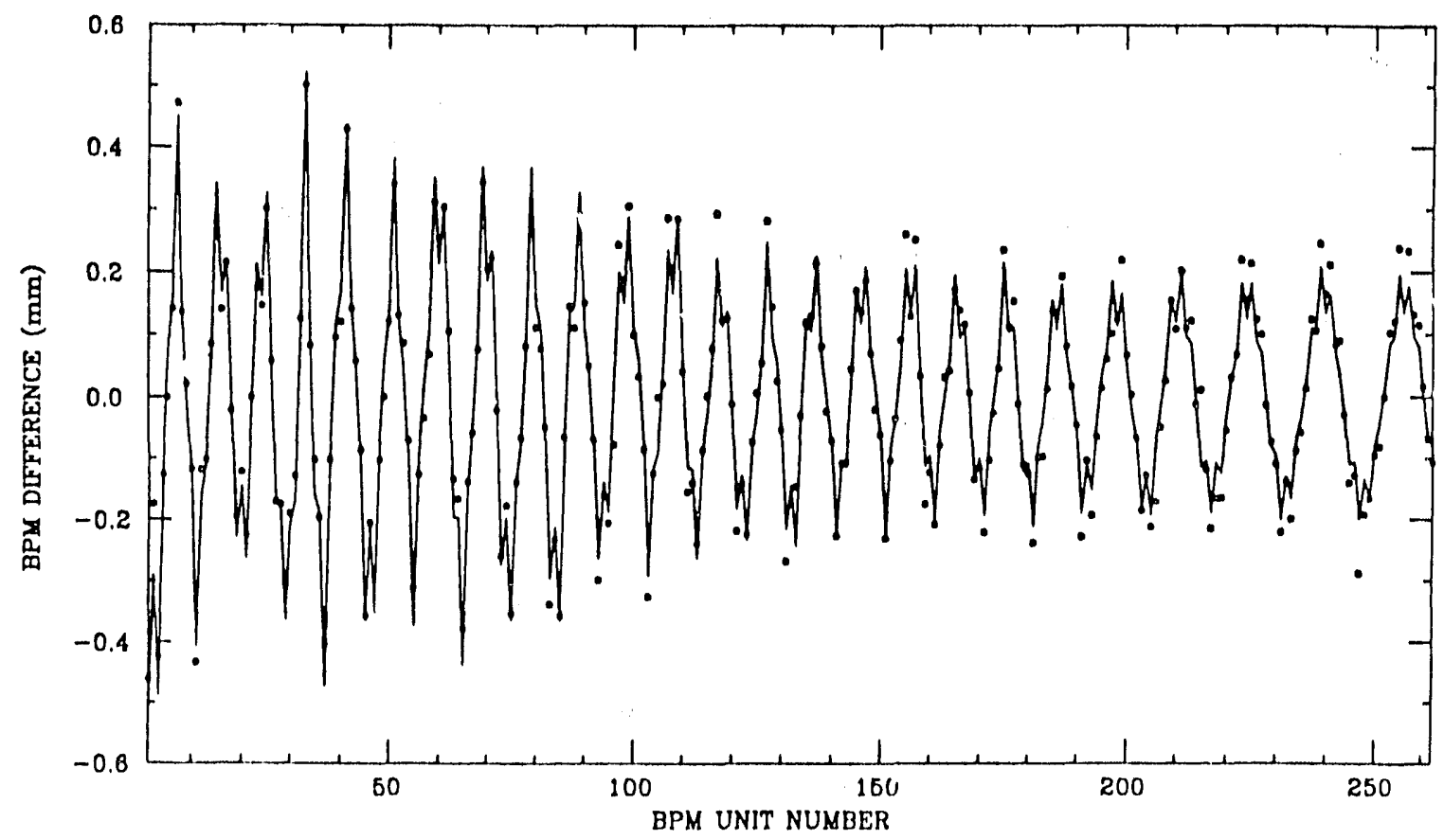

Figure 17 An observed oscillation (dots) is fit to a betatron oscillation (solid line) using the equation of motion with a single adjustable energy parameter over the whole linac. Transverse wakefields in the SLC produce apparent beam energy errors of a few percent. 


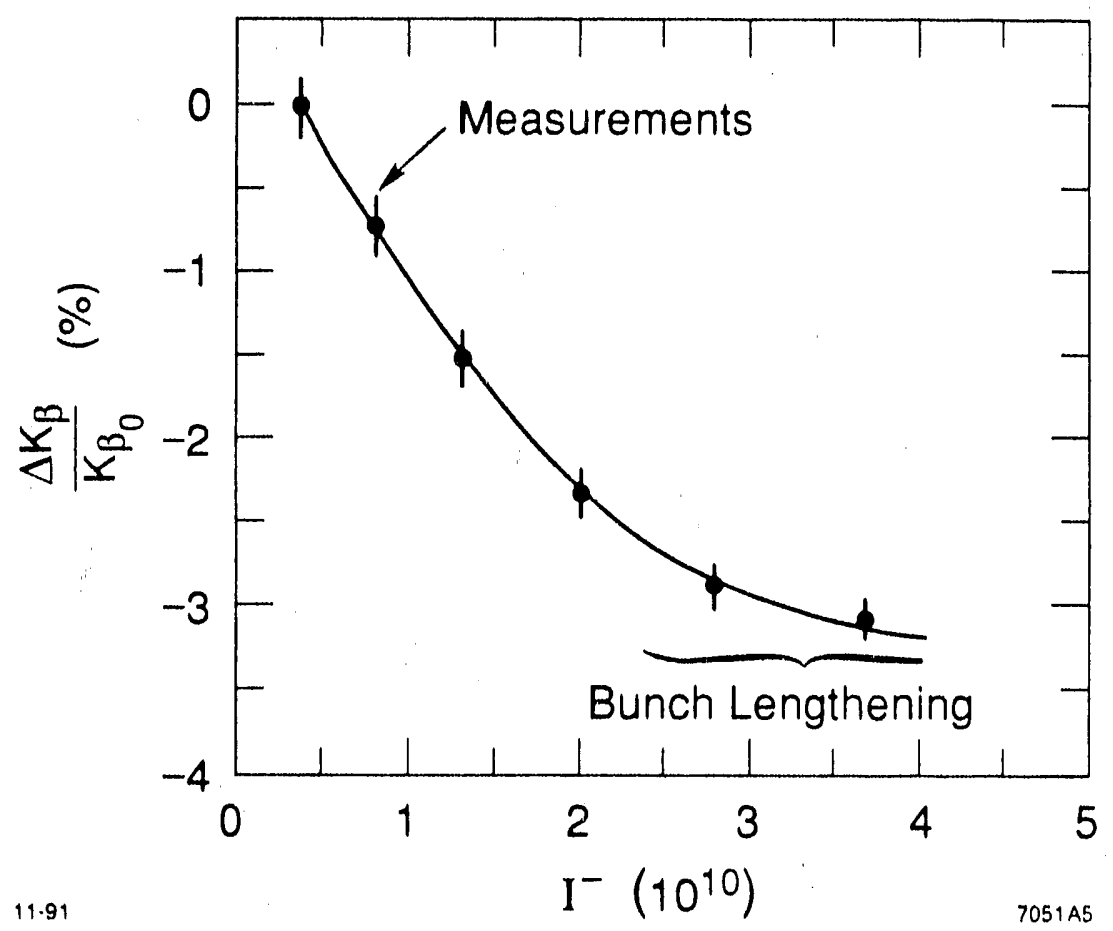

Figure 18 Change of the measured betatron spatial frequency due to transverse wakefields as a function of beam current in the SLC. These wakefields retard apparent betatron oscillations.

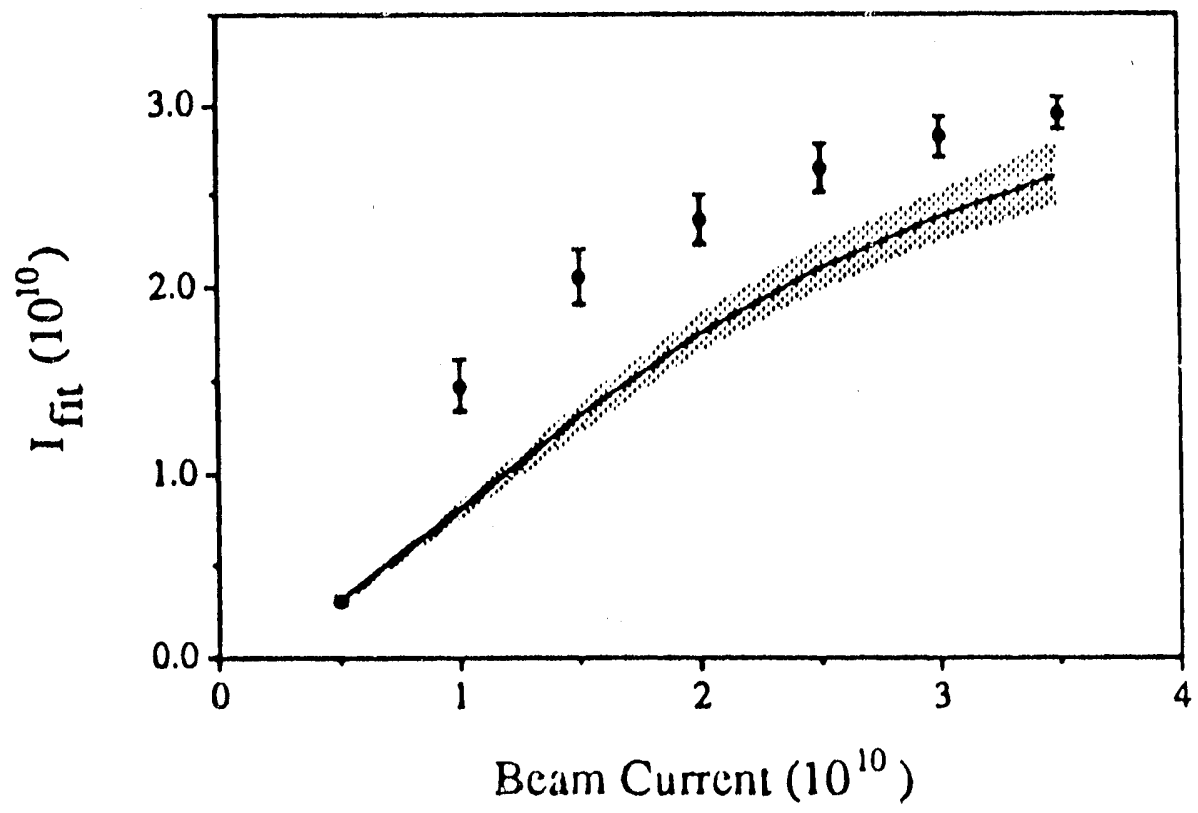

Figure 19 The fit value for the beam current from measured (circles) and simulated (solid lines) BPM difference trajectories as a function of the actual beam current in the SLC. The measured and simulated trajectories were fit with the same algorithm. The curves show that even at relatively low bunch intensities the transverse wakefield effects influence the trajectories. The differences between the measurements and simulations arise from the uncertainty of the longitudinal bunch profile, the incoming beam transverse profile, and an uncertainty in the transverse wake potential. 
A forty-one slice simulation of autophasing for the SLC has been done [8]. Some of the results are shown in Figures 21 and 22. The difficulties in achieving the conditions of Equation 12 are apparent as Plots 21c and 21e must be matched over their full width. The irregularities of the lattice, the strong curvature of the RF voltage, and the need for a small energy spread at the end of the linac have made practical autophasing not fruitful to date, although studies continue. Autophasing without acceleration has also been studied [21]. Nevertheless, if this condition can be satisfied in a future collider, the beam intensity threshold for wakefield emittance growth will increase significantly .

\subsection{Injection Tolerance}

The tolerance on injection parameters can be calculated with several goals in mind. One goal is to have the emittance of the beam not increase unacceptably during acceleration.

$$
\Delta \varepsilon / \varepsilon<0.1
$$

Another goal might be to keep the transverse position of the beam at the end of the accelerator within some fraction of the beam size from the accelerator axis to maintain luminosity.

$$
\Delta \mathrm{x}<\sigma_{\mathrm{x}} / 3
$$

Conversely, the goal may be to limit the number of particles a certain distance from the axis, as if to protect a device from destruction (i.e. collimator).

$$
\int_{d}^{\infty} \rho(x) d x \leq n
$$

where $\rho$ is the particle density and $d$ is the off axis distance of interest.

A tolerance on injection position (and angle) jitter can be calculated for the SLC from the data in Figure 15, with proper BNS conditions at each current. To maintain luminosity using Equation 14 , the oscillation must be kept below about $\sigma_{x, y} / 3$ at the end of the linac. The results of these calculations are shown in Figure 23 [22]. At $7.5 \times 10^{10}$ particles, the displacement at the beginning of the linac must be less that $40 \mu \mathrm{m}$, within a factor of two of present operational values. Similar tolerances for a future collider will be significantly smaller, as the emittances are much lower.

\subsection{Transverse jitter}

Feedback systems that work pulse-by-pulse are essential for kecping the beam parameters within acceptable limits given the many possible sources of transverse jitter. Several sources are varying power supplies of dipoles and off axis quadrupoles, vibrating quadrupoles, klystron phase and amplitude jitter, 


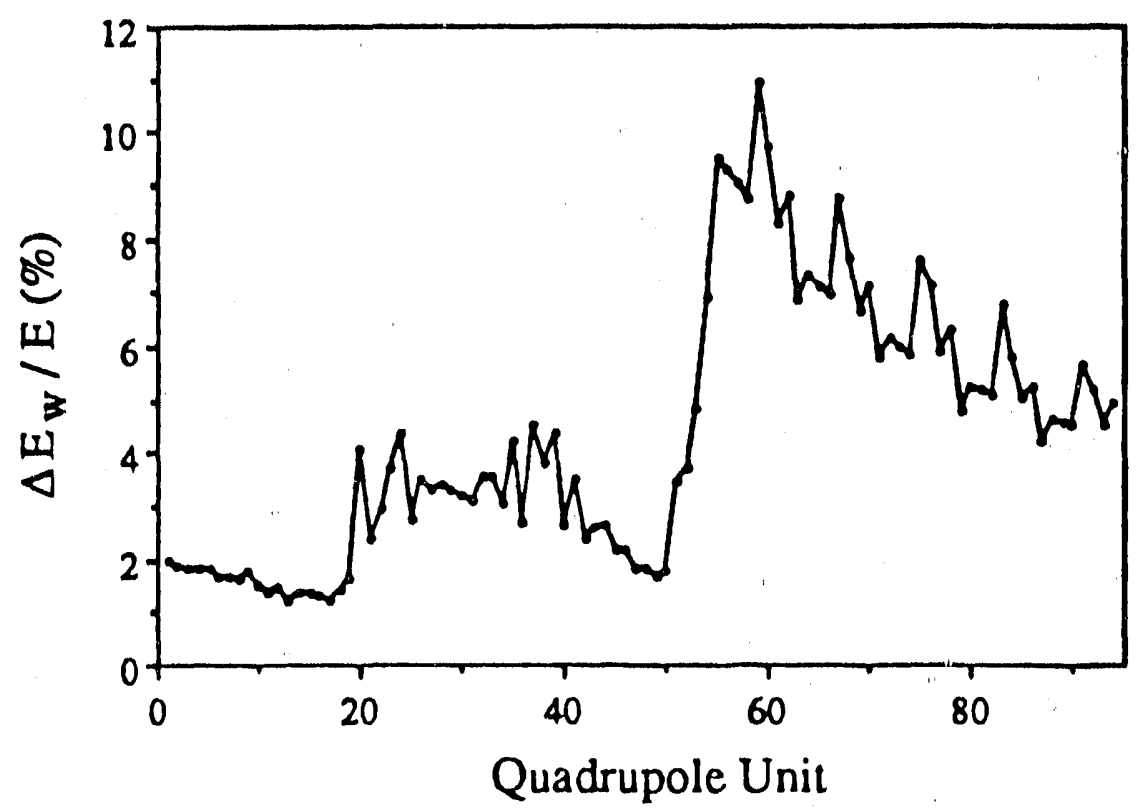

Figure 20 An equivalent energy change at the back of the bunch which cancels the phase advance per cell from transverse wakefields at $3 \times 10^{10}$ electrons plotted versus quadrupole unit number resulting from a two particle model. The variations result from the uneven spacing of the quadrupoles. Adjustments in the energy spectrum using klystron phases can not be induced in the beam as rapidly as the discontinuities require. However, the general trends can be followed.
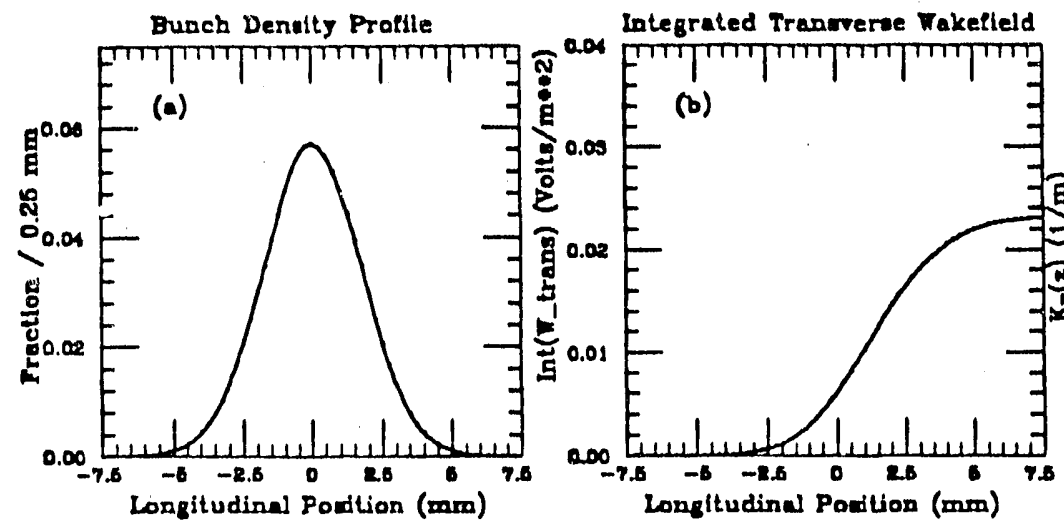

Required Latlice $\mathbf{X}(\mathbf{x})$ from Wake:

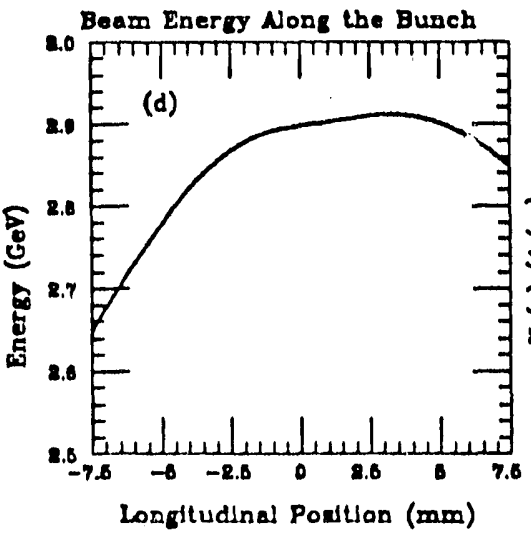

Lattice $K(z)$ from Ineray and Quad.

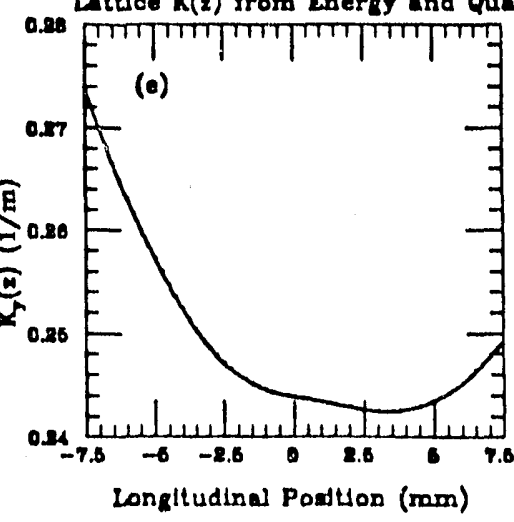

Figure 21 Schematic representation of the mutual compensation of wakefield and chromatic effects over the entire bunch in the SLC linac. The bunch density is shown in (a). The integrated transverse wakefield over the bunch is shown in (b). The required betatron frequency determined by transverse wakefields over the bunch is shown in (c). The energy is shown in (d). Finally, the betatron frequency along the bunch as determined from lattice and bunch energy considerations is shown in (e). The task for autophasing is to match (c) with (e) by adjusting the energy spectrum, bunch length, and lattice parameters. 

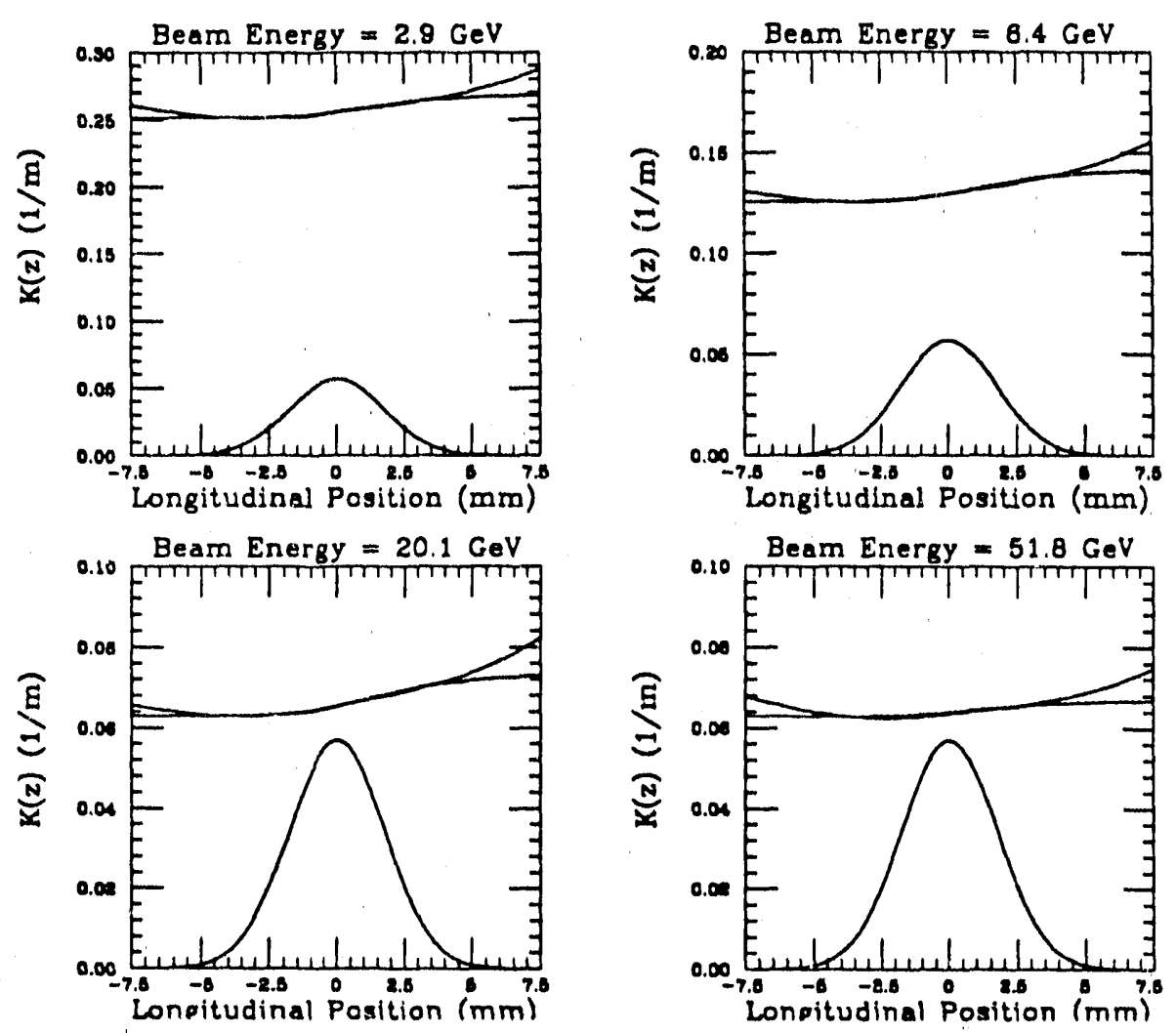

Figure 22

An optimized autophasing condition is shown at four locations along the SLC where the wakefield required and lattice generated betatron frequencies are superimposed over the length of the bunch. In this solution the klystron phases are specially set to $-7^{0},-10^{0}$, $-20^{0},-1^{0}$, and $0^{0}$ for five linac sections of length $100 \mathrm{~m}, 200 \mathrm{~m}, 400 \mathrm{~m}, 800 \mathrm{~m}$, and $1400 \mathrm{~m}$, respectively. The resulting matches are reasonably good over the length of the bunch. The bunch charge is $3.5 \times 10^{10}$ electrons with a length of $1.75 \mathrm{~mm}$ in a 90 degrees per cell lattice. The final energy spread is $1.4 \%$. which is too large for the SLC final focus to chromatically correct. By adjusting the phase of the last section of the linac, the energy spread can be reduced $b$ " the auto phasing condition is then violated leading to emittance growth.

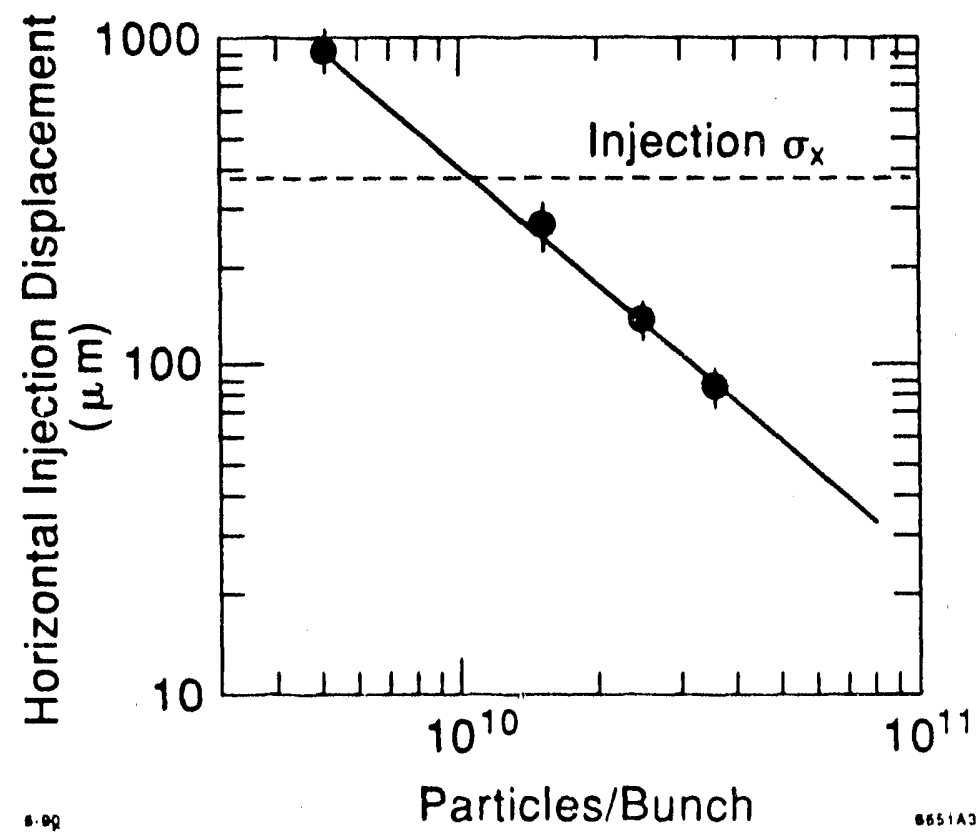

Figure 23 Injection tolerances versus bunch population as determined for the SLC using the data in Figure 14 and the requirement that the oscillation be smaller than the final bunch size. 
dark current in the RF structure, unstable kicker magnets, temperature changes in the tunnel [23], and changes in the beam intensity. In the SLC, over 80 beam parameters (beam positions, angles, and energies) are controlled by feedback routinely with many corrections each second. Not all parameters need rapid feedback. For example, the energy spectrum feedback has proven not to need pulse-by-pulse control. Modern control theory is used to provide cascaded control of position and angle loops from the beginning to the end of the accelerator that minimally interfere with each other and provide maximum control [24]. Reduction of oscillations with frequencies up to one sixth of the accelerator pulse rate can be expected.

Injection jitter too rapid for feedback control must be isolated and fixed. The sources of jitter are generally not stable, as can be seen in Figure 24. Thus, sophisticated analysis techniques must be developed.

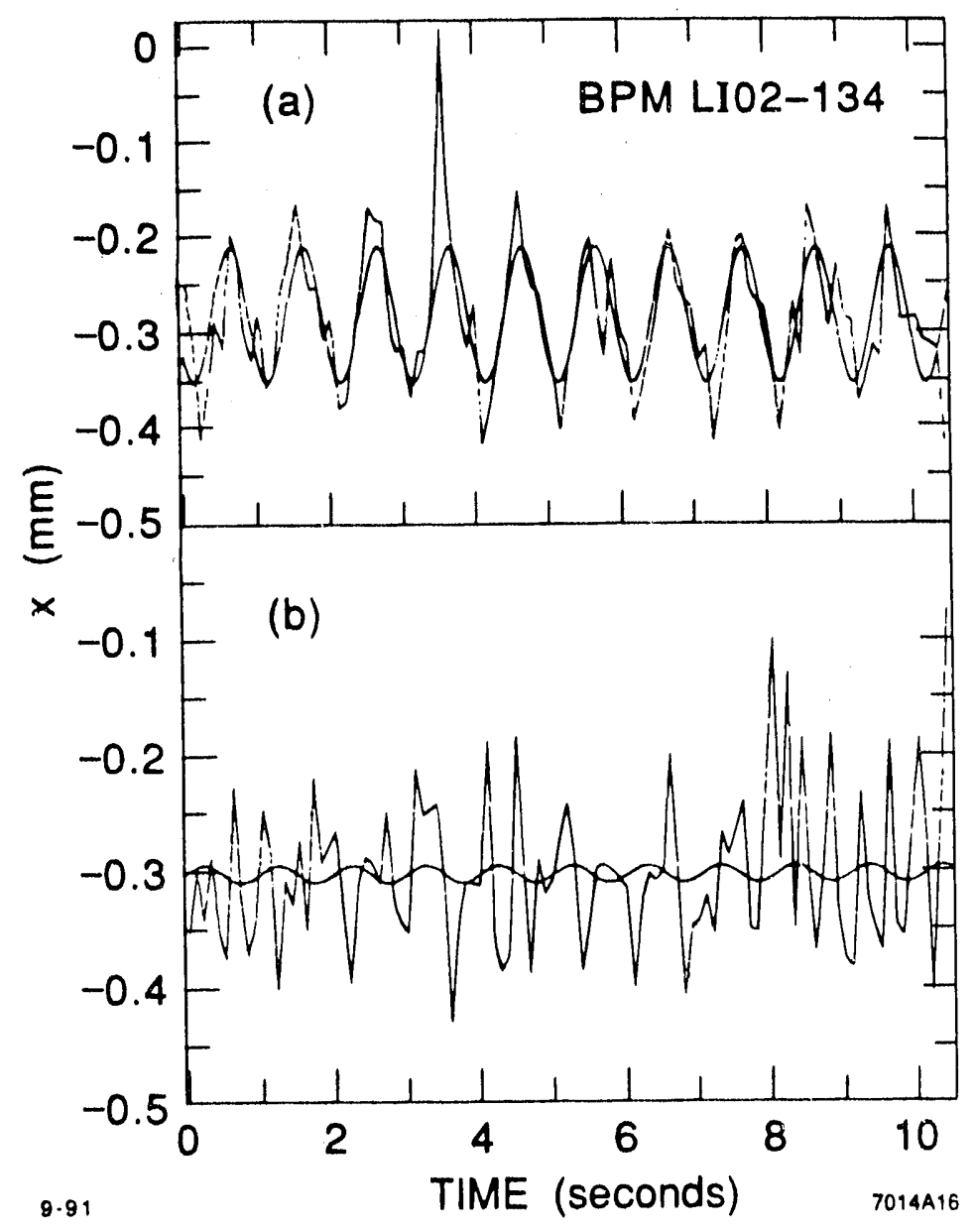

Figure 24 Measured bunch position in the early SLC as a function of time (straight lines) for two periods separated by 3 minutes. The sine curve is a fit to the data at $1.2 \mathrm{~Hz}$. Note that the frequency spectrum of the beam jitter can change rapidly, even though the root-mean-square jitter is about the same.

The magnitude of the jitter can depend strongly on current. An observation in the SLC is shown in Figure 25 where the horizontal jitter becomes rapidly stronger above $3.5 \times 10^{10} \mathrm{e}^{-}$per pulse. The nature of this jitter can be scen in Figure 26 and 27. The probable cause for this rapid change is the large dispersion 


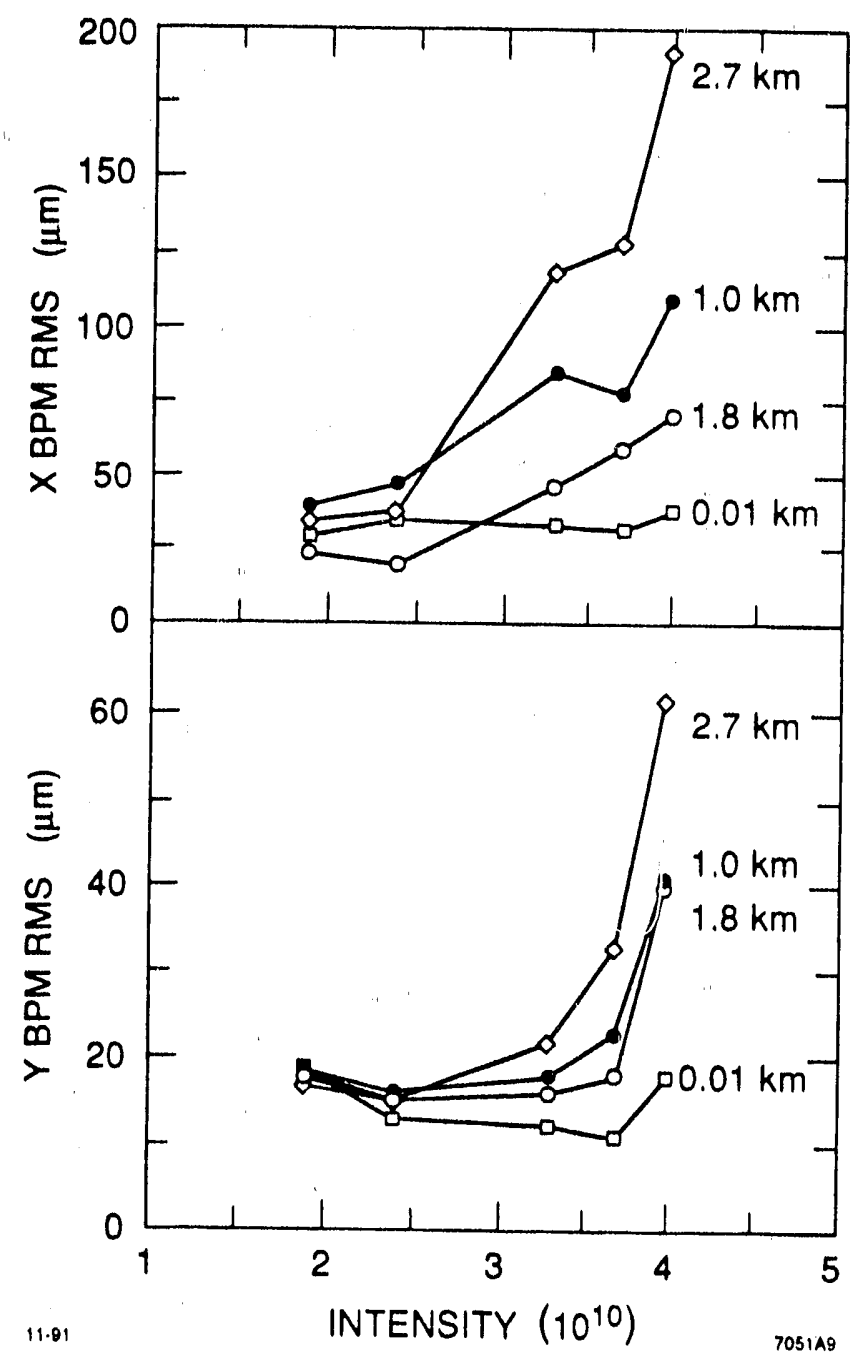

Figure 25 Measured transverse position jitter in the SLC beam as a function of current and position along the linac. At low currents, all positions have similar root-mean-square jitter values. However, at high currents the downstream locations indicate much stronger oscillations. Studies suggest that the injected beam develops a quadratic offset profile at high currents which causes an increased jitter in the linac.

in the transport line between the damping ring and the linac combined with the lengthened bunch in the damping ring at high currents. At the resulting large amplitudes the particles at the front and back are focused incorrectly. These particles then enter the linac far off axis and far ahead or behind the main core of the beam. The head particles excite wakefields downstream $[25,26]$. Correction of the second-order optics in the transport line is warranted.

If a bunch has a skew profile as it enters the accelerator, it is not possible to correct the bunch with steering to remove all transverse wakefield effects. Some of the possible injection conditions (errors) of a bunch into an accelerator are shown in Figure 28, indicating the importance of a well corrected and controlled launch. Plot (a) is for a perfect bunch, (b) shows a transverse offset, (c) shows a head-tail energy spread and dispersion $\eta$, (d) indicates upstream wakefields, (e) characterizes second order terms ( $\mathrm{T}_{166}$ for example), and (f) is similar to (c) but with $\eta^{\prime}$ added.

Dispersion effects will be discussed in more detail. Dispersion at the entrance to the accelerator can cause the beam to have a transverse-longitudinal tilt, similar in shape to Figure $28 \mathrm{c} . \Delta \mathrm{x}=\eta \Delta \mathrm{E} / \mathrm{E}$. The 


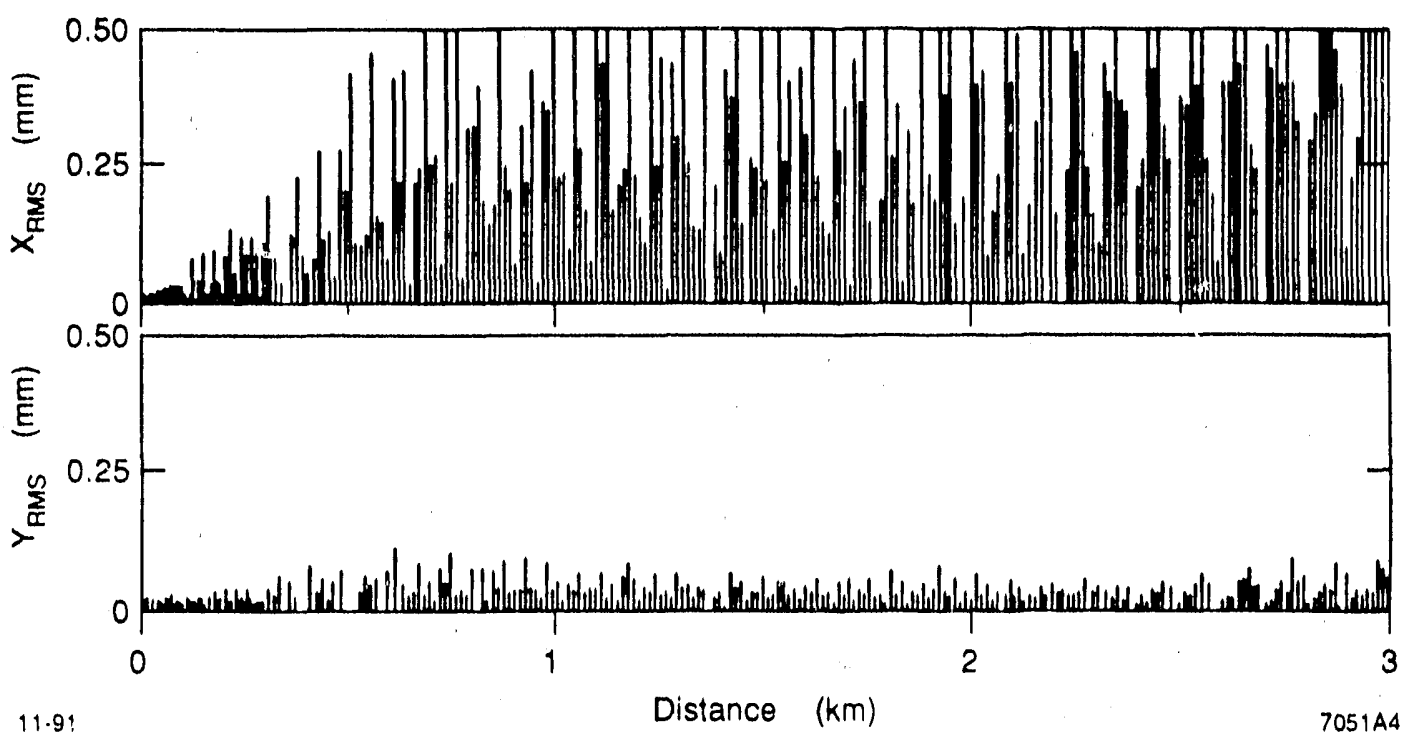

Figure 26 The rms trajectory averaged over 10 pulses is shown along the SLC linac for $4 \times 10^{10} \mathrm{e}$. The jitter causes the $\mathrm{x}$ amplitude to grow rapidly. The $\mathrm{y}$ enlargement is acceptably small.
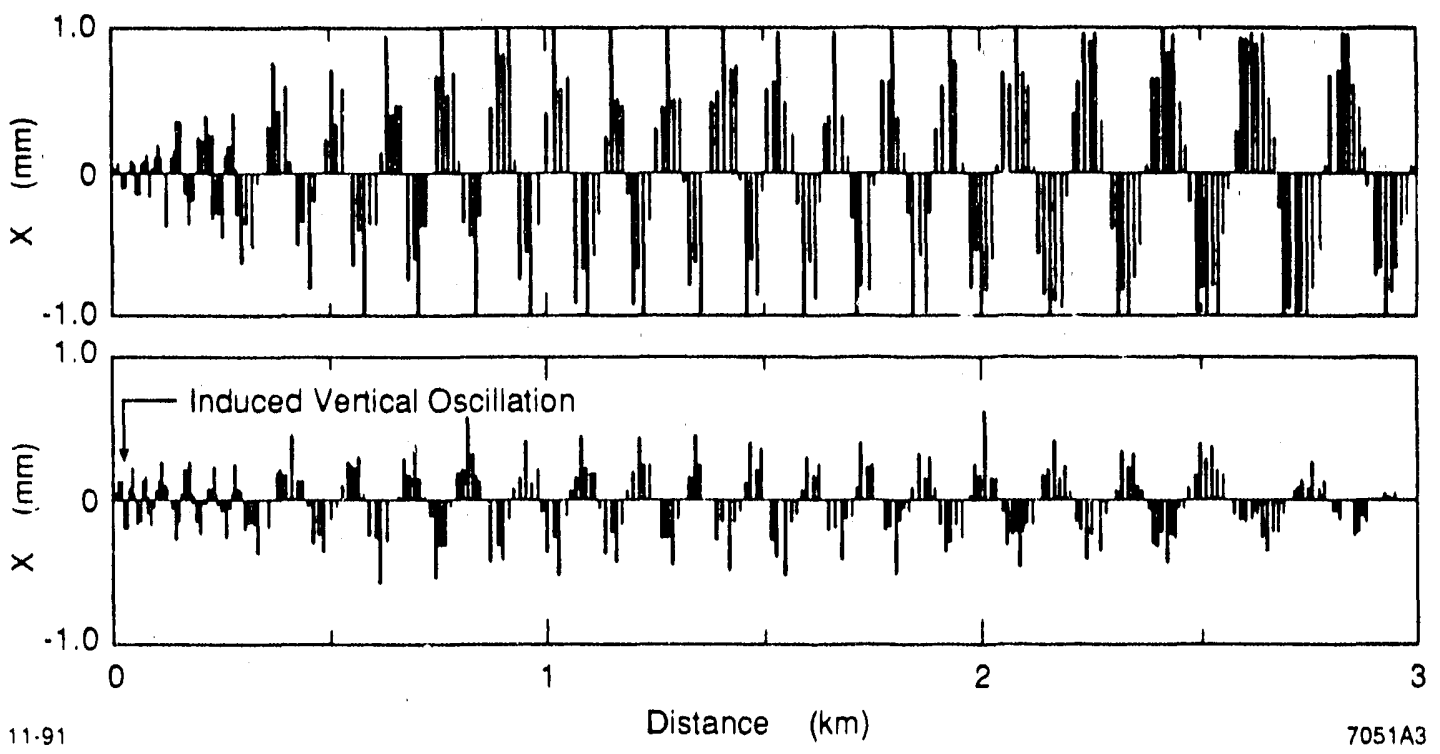

Figure 27 A horizontal oscillation resulting from the unknown jitter shown in Figure 25 and 26 at $4 x$ $10^{10} \mathrm{e}$ - in the SLC. A vertical oscillation was also produced but with a change in a dipole magnet early in the linac. The vertical growth is not the same as the horizontal, indicating that the transverse offsets over the length of the bunch are not like those from a dipole deflection but more likely a higher moment. A possible configuration has the core on axis but the head and tail off axis quadratically.
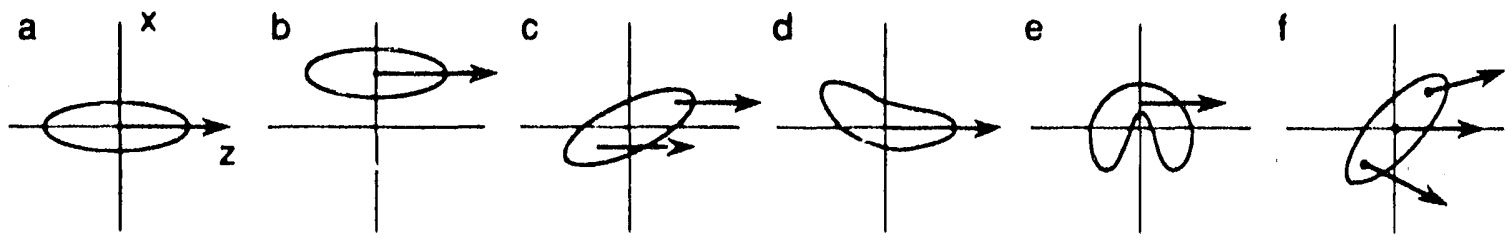

9-91

$7014 A 10$

Figure 28 Poscible injection orientations of a bunch entering the linac. All orientations have wakefield and dispersion implications except for plot (a), the idcal condition. 
head of the bunch is not on axis even though the average positicn of the bunch is. The oscillation of the head downstream will most likely drive the trailing portions of the bunch to large amplitudes, increasing the emittance. Calculations of this effect for the SLC linac give a tolerance on the allowed dispersion as a function of current [27]. The results are shown in Figure 29. Besides the removal of the dispersion errors, careful placement of the bunch and the use of BNS damping are quite helpful. However, jitter in the energy of the beam entering the linac can make this problem especially troublesome.

The RF structures in the linac have several small asymmetries that generate transverse fields [28]. These transverse fields can be in- or out-of-phase with the accelerating fields and deflect (slightly) all or portions of each bunch, as can be seen in Figure 30. The accumulation of these kicks along the linac can enlarge the emittance. There are three effects. (a) The average deflection component can be removed by trajectory correction. However, changes in the RF phase or amplitude of a klystron cause changes in the deflecting fields and thus generate position jitter downstream [29]. (b) The steady state portion of the transverse RF kick causes local trajectory changes, even with steering, and produces off center beams leading to dispersion and wakefield growth [30]. (c) Finally, the magnitude of an RF deflection can vary over the length of the bunch, which causes direct emittance growth and further filamentation downstream [31]. Care must be taken during construction of the RF structure to minimize these asymmetries. They cannot be fixed after construction except possibly by cancellation using appropriate pairing.

\section{Transverse Emittance Effects At High Currents}

\subsection{General emittance reduction techniques}

In a linear accelerator many emittance enlargement effects may occur: some result from injection errors, some arise from accumulation of errors along the linac, and some occur at the end. The injection errors include betatron mismatches, dispersion mismatches, $x-y$ coupling, static injection offsets, and launch jitter. Accumulating errors include transverse wakefields, misaligned quadrupoles and position monitors, RF deflections, and component vibration. Effects at the end of the linac include collimator wakefields and $x-y$ coupling.

Betatron mismatches occur when the injection beam has a phase-space orientation $(\beta, \alpha)$ that does not match the linac lattice. Because of geometry the linac lattice cannot be chromatically corrected. Therefore, particles with different energies have different oscillation frequencies. Since the injected beam has an internal energy spread that changes during acceleration, the trajectories of the different energy portions of the beam rotate in phase space at different speeds and soon undergo filamentation. Given a beam $\beta_{b}$ and $\alpha_{b}$ that are mismatched from the lattice design values $\beta_{1}$ and $\alpha_{1}$, the emittance enlargement after filamentation is given by a parameter Bmag [32].

$$
(\gamma \varepsilon)_{\text {final }}=\operatorname{Bmag}(\gamma \varepsilon)_{\text {initial }}
$$




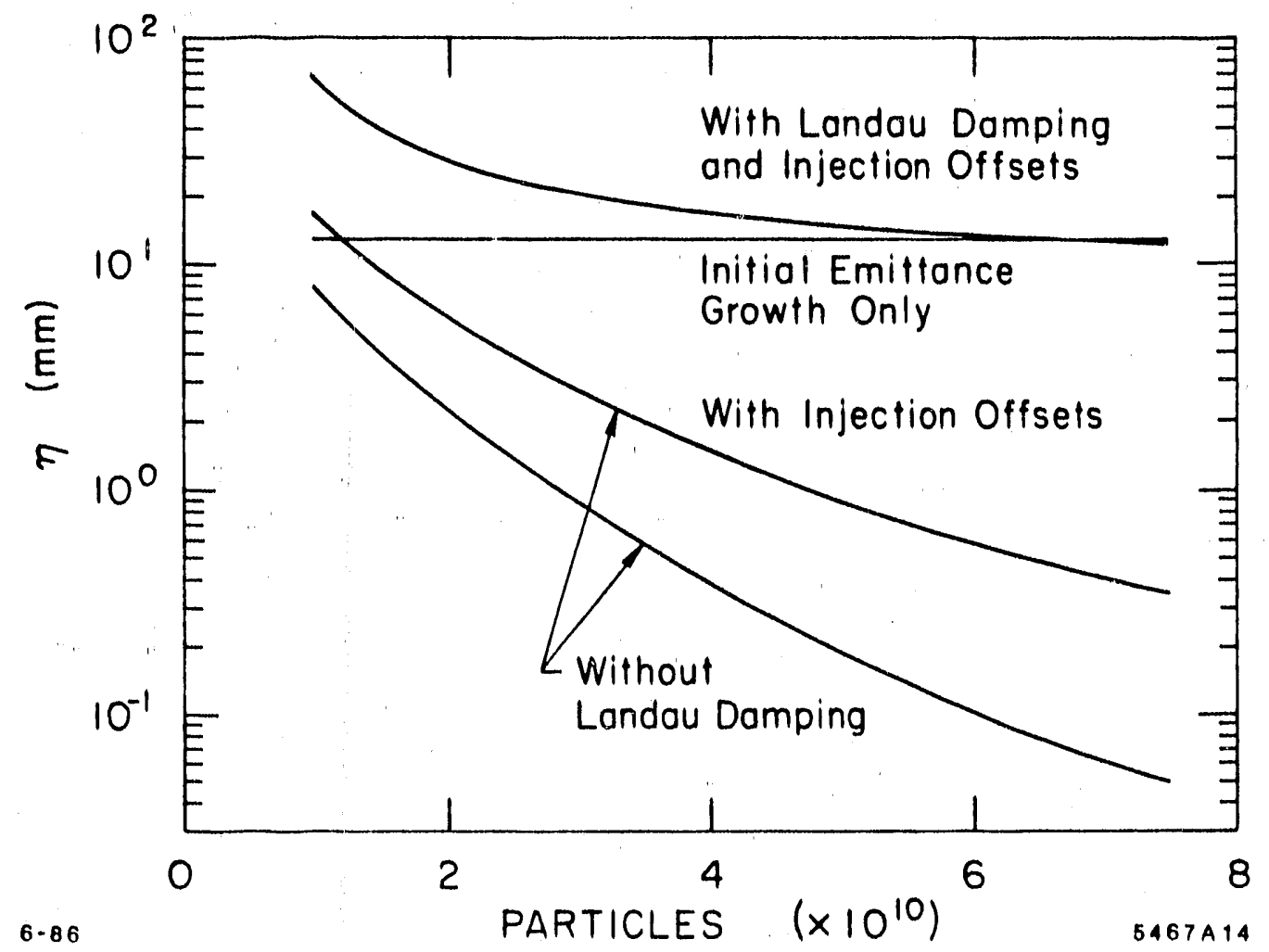

Figure 29 Upper limits of the allowed residual dispersion $\eta$ entering the SLC linac as a function of bunch intensity. The limits are calculated for a partially compressed bunch $(1.5 \mathrm{~mm})$ where dispersion tilts the bunch transversely and excites transverse wakefields in the linac. Steering the head of the beam near the axis increases the tolerances. Also, BNS damping (Landau damping) increases the tolerances significantly. Higher order dispersion will have equivalent effects.

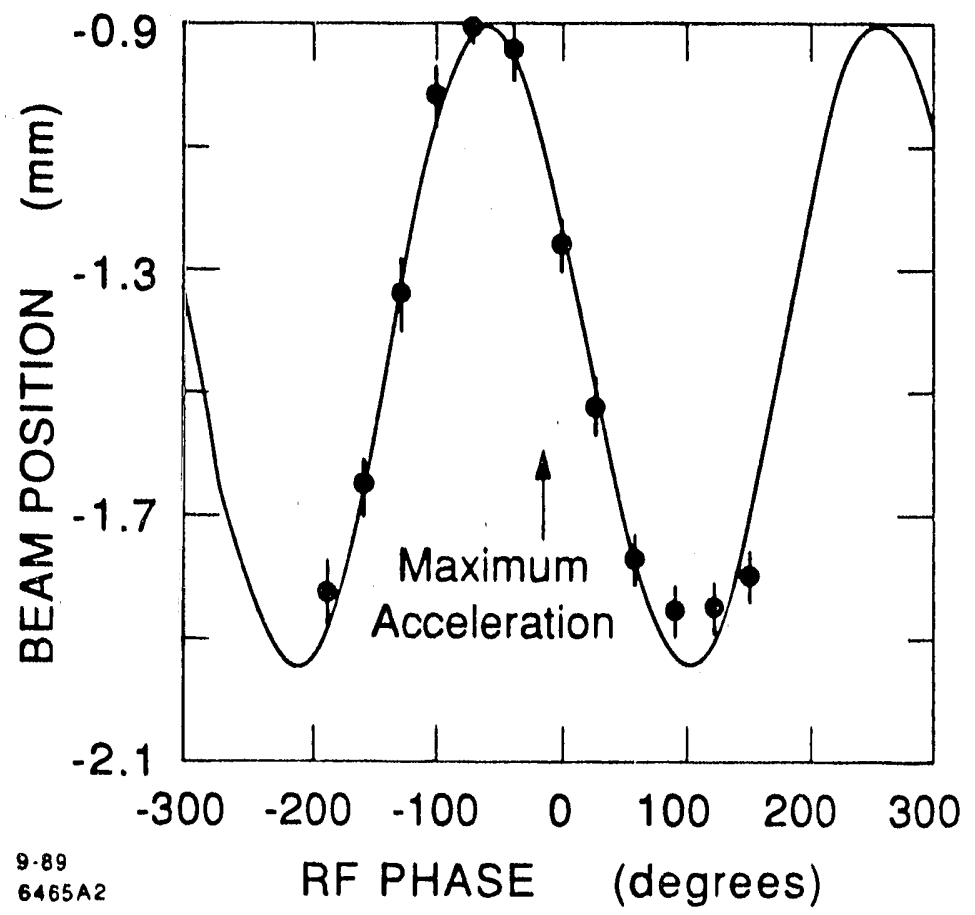

Figure 30 Measured RF deflections of a beam in the SLAC linac are seen as position changes downstream with phase changes. The beam energy was $1 \mathrm{GeV}$ and the accelerating section with the changing phase was $12 \mathrm{~m}$ long with an accelerating gradient of $12 \mathrm{MeV} / \mathrm{m}$. There are several consequences. Jitter in the phase and amplitude of the klystrons produces dipole deflections in the beam. The finite longitudinal length of the beam results in the beam shearing transversely from the RF deflection and having an increased emittance. 
with

$$
B m a g=\frac{1}{2}\left[\frac{\beta_{1}}{\beta_{b}}+\frac{\beta_{b}}{\beta_{1}}+\beta_{b} \beta_{1}\left(\frac{\alpha_{b}}{\beta_{b}}-\frac{\alpha_{1}}{\beta_{1}}\right)^{2}\right]
$$

To avoid emittance increases of order $10 \%, \beta$ must be matched to $30 \%$ or so. The $\alpha$ match has a similar constraint.

Dispersion mismatches are similar [33,34]. A dispersion $\eta$ is a transverse position-energy correlation which adds to the apparent size and emittance of the beam: $\sigma^{2}=\varepsilon \beta+\eta^{2}(\Delta E / E)^{2}$. Given the chromatic lattice, the beam particles displaced by dispersion undergc filamentation if allowed and the real emittance grows. The measured effective emittance $\varepsilon_{\mathrm{eff}}$ in the presence of dispersion [32] is given by

$$
\varepsilon_{\mathrm{eff}}^{2}=\varepsilon_{\beta}^{2}+\frac{\varepsilon_{\beta}}{\beta_{0}}\left[\eta^{2}+\left(\beta_{0} \eta^{\prime}+\alpha_{0} \eta\right)^{2}\right]\left\langle\delta^{2}\right\rangle
$$

and with filamentation the emittance increases further leading to a final value of

$$
\varepsilon_{\mathrm{eff}}=\varepsilon_{\beta}+\frac{1}{2 \beta_{0}}\left[\eta^{2}+\left(\beta_{0} \eta^{\prime}+\alpha_{0} \eta\right)^{2}\right]\left\langle\delta^{2}\right\rangle .
$$

The errors in the dispersion on injection must usually be controlled to a few millimeters. Minimization of the measured emittance early in the linac is made by adjusting quadrupoles in a dispersive region upstream to remove the dispersion errors [35]. Future colliders require an order of magnitude smaller adjustments.

Emittance growth from $x-y$ coupling occurs when particle trajectories in one plane, say $x$, are rotated into the other plane $(y)$ by skew transport elements such as rotated quadrupoles or off-axis sextupoles. This problem is of more concern for a future collider, where flat beams are needed. Coupling can increase both effective emittances ( $x$ and $y$ ) or move emittance from one plane to the other [36]. Filamentation of the mixed beam can cause further grow th.

\subsection{Transverse profile changes with transverse wakefields}

A betatron oscillation in the linac combined with transverse wakefields leads to the potential for strong wakefield enlargement. Studies of beam transverse enlargement at the end of the SLC linac from betatron oscillations generated by a dipole magnet are shown in Figure 31. These measurements were made with profile monitors using fluorescent screens and TV cameras producing images as shown in Figure 32. The observations in Figure 31 clearly show that leading particles which are off-axis in the accelerator excite trailing particles to larger amplitudes. It is evident that the transverse beam tails oscillate along the linac at the betatron frequency.

The effects discussed in Section 4.1 which affect the transverse beam profile are symmetrical, leaving the beam with left-right equality. Transverse wakefields, however, are asymmetrical and often produce skewed distributions. The particle distributions resulting from wakefields are not gaussian and 

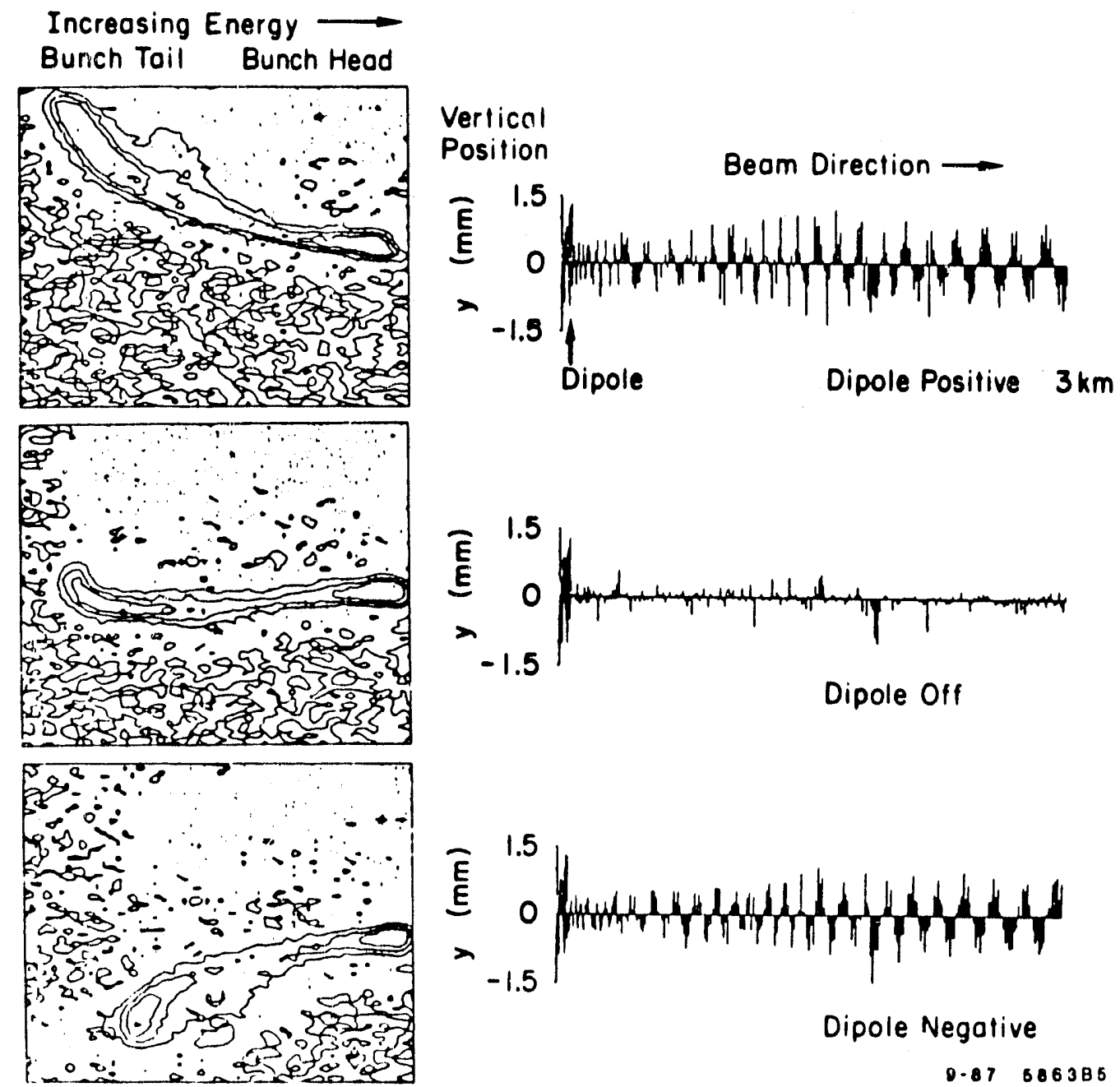

Figure 31 Experimental results demonstrating that the bunch head drives the tail to larger amplitudes during a betatron oscillation due to transverse wakefields. On the right side three trajectories (transverse positions along the linac) are shown. The upper plot has a 'positive' oscillation produced by a small change in a dipole magnet early in the linac, the center shows 'no' oscillation, and the bottom a 'negative' oscillation. On the left side the three resulting beam shapes are shown measured on a profile monitor. The accelerator conditions are such that the longitudinal head of the bunch is located on the right side of the measured profile and the longitudinal tail is on the left side. Note that the position of the bunch head is independent of the oscillation but that the tail of the bunch moves vertically with the sign of the oscillation. This example shows that leading particles in a bunch drive trailing particles to large amplitudes via transverse wakefields. It also shows that the phase space dimensions of each longitudinal slice of the beam remairs the same even though the effective emittance of the beam as a whole has increased from transverse wakeficld effects. 


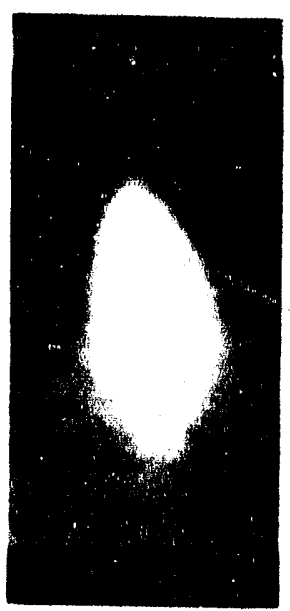

9-89
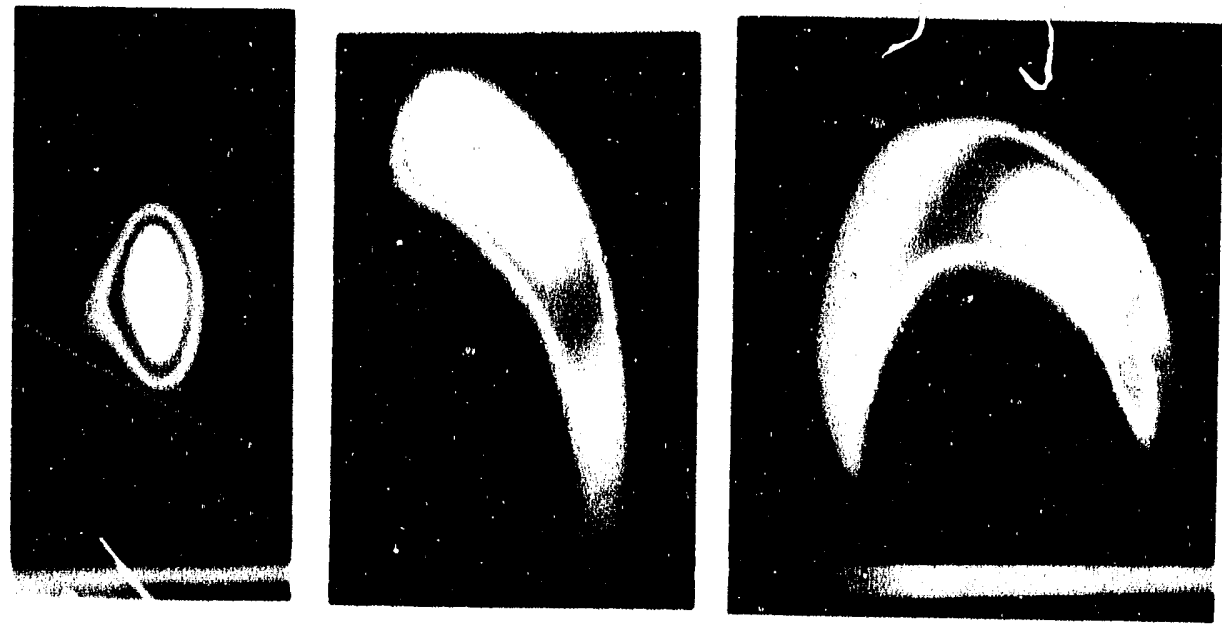

643846

Figure 32 Images of an electron bunch on a profile monitor at $47 \mathrm{GeV}$ showing wakefield growth with increasing oscillation amplitudes. The images from left to right are for a well-steered beam, a $0.2 \mathrm{~mm}$ oscillation, a $0.5 \mathrm{~mm}$ oscillation, and a $1.0 \mathrm{~mm}$ oscillation, respectively. Both horizontal and vertical oscillations are present. The beam intensity is $2 \times 10^{10}$ electrons. The core sizes $\sigma_{\mathrm{X}}$ and $\sigma_{\mathrm{y}}$ are about $120 \mathrm{~mm}$.

must be described in a new way. Special methods are available to model and control these changes [38]. Multiple gaussian fits, skewness, kurtosis, and fitting left and right halves separately have been tried with various degrees of success. An intuitive accelerator physics model for tail propagation is described here.

A bunch executing a betatron oscillation in the quadrupole lattice of the linac experiences transverse wakefields in the accelerating structure. The head of the bunch drives the core and back of the bunch to ever increasing amplitudes producing a non-gaussian tail. Simulations of this growth have been made where a bunch is divided into longitudinal slices and traced through the linac. A nearly exponential growth from head to back is apparent. The transverse particle distribution $\rho(x)$ of each slice is equal to that of the initial phase space distribution. The initial particle distribution is a gaussian with width $\sigma$.

$$
\rho(x)=\exp \left(-\left[x-x_{0}\right]^{2} / 2 \sigma^{2}\right) / \sigma(2 \pi)^{1 / 2}
$$

where $x_{0}$ is the mean of the distribution. The overall distribution must be integrated over the slices with different transverse positions $x_{0}$. The position $x_{0}$ of each slice is represented by an exponential which initiates at position $z 0$ along the length of the bunch and has a growth distance of $\tau$. This tail rotates in phase space with the betatron phase $\phi_{i}$ but has an initial phase $\phi_{0}$. The emittance of each slice of the beam is $\varepsilon$ and the betatron function at each profile measurement ' $i$ ' is $\beta_{\mathrm{i}} . \sigma_{\mathrm{i}}{ }^{2}=\varepsilon \beta_{\mathrm{i}}$. The tail extension is scaled locally by $\sigma_{\mathrm{i}}$.

$$
x_{0}\left(\phi_{i}, z\right)=\sigma_{i} U(z 0-z)\left[\exp \left((z 0-z) \tau / \sigma_{z}\right)-1\right] \cos \left(\phi_{i}+\phi_{0}\right)
$$


where $U$ is the unit step function and the bunch length is $\sigma_{z}$. The transverse distribution of each slice is given by :

$$
\rho\left(x, \phi_{i}, z\right)=\exp \left(-\left(x-x_{0}\left(\phi_{i}, z\right)\right)^{2} / 2 \sigma_{i}^{2}\right) /(2 \pi)^{1 / 2} \sigma_{i}
$$

Finally, the overall transverse distribution called $f\left(x, \phi_{i}\right)$ is given by

$$
f\left(x, \phi_{i}\right)=\int_{-\infty}^{+\infty} \rho\left(x, \phi_{i}, z\right) h(z) d z
$$

where $h(z)$ is the longitudinal profile, usually assumed to be a gaussian with length $\sigma_{z}$. By choosing $\phi_{0}$, $\tau$, and $z_{0}$, the beam shape can be calculated at any location over a reasonably short region of the linac (less than several betatron wavelengths so that wakefields do not change the distributions). Measured beam shapes can be analyzed to determine the tail structure of the beam and measure the effective $\phi_{0}, z_{0}$, and $\tau$. For a test, an oscillation was induced in the SLC electron beam with a charge of about $2.5 \times 10^{10} \mathrm{e}^{-}$by a dipole magnet and the resulting trajectory is shown in Figure 33. The associated beam profiles on four wire scanners are shown in Figure 34. The observed beam shapes have a definite tail with a particular phase, readily fit with the above algorithm.

This model assumes that the transverse tails are not large. As an experiment, an SLC beam was lengthened to about $2.5 \mathrm{~mm}$ and accelerated. The expected transverse wakefield effects were large. The internal energy spread was also exaggerated. The resulting beam profile is shown in Figure 35. Clearly, a full model of all parameters must be used to diagnose beams with distributions like the one in Figure 35.

\subsection{Emittance observations}

The emittance of the besm at injection into the linac is important as it gives the scale of the tolerances to be studied. For example, the measured emittances at the entrance to the SLC linac are shown in Figure 36. The horizontal emittance increases with intensity because of bunch lengthening with current in the damping ring $[12,39]$.

The beam launch parameters into the linac must be correct in order to avoid filamentation and wakefield effects downstream. To repeat, the dispersion functions must be properly adjusted, the betatron mismatches removed, and the second-order optics checked. Operation and studies of the SLC have shown that these emittance enlargement effects including chromatic and wakefields effects can be controlled during colliding beam operations to provide significant luminosity [40]. The best measured single bunch emittance conditions of the SLC to date are shown in Figure 37. These results were also obtained during collisions with three bunch operation (e+,e-, e- scavenger) up to $3 \times 10^{10}$ particles per bunch. The extension of these techniques to higher currents is now under active study as intensities near $4.5 \times 10^{10}$ are needed and the excessive horizontal beam jitter at high currents mentioned in Section 3.6 must be reduced. 


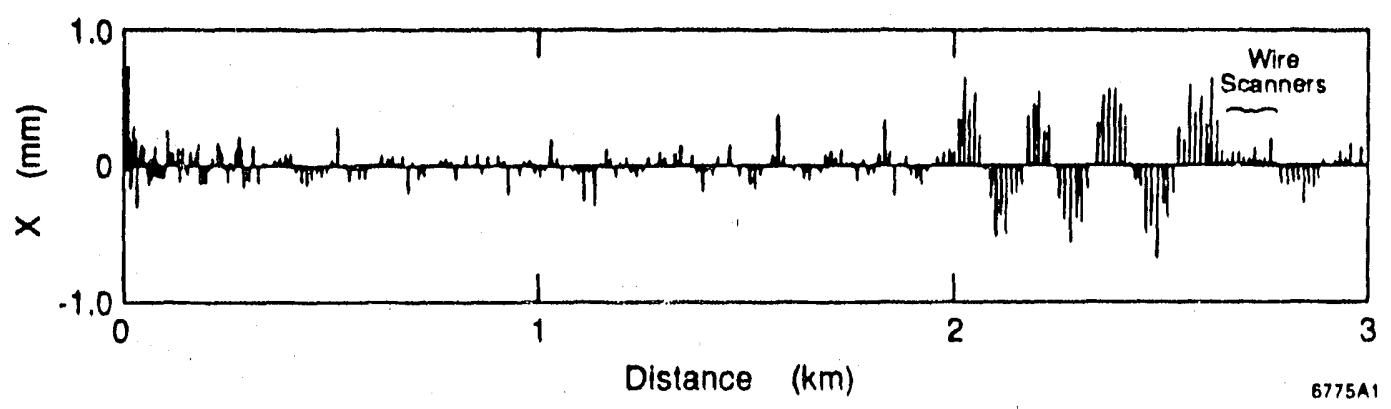

Figure 33. A particular beam oscillation used to generate the transverse beam tails displayed in Figure 34.

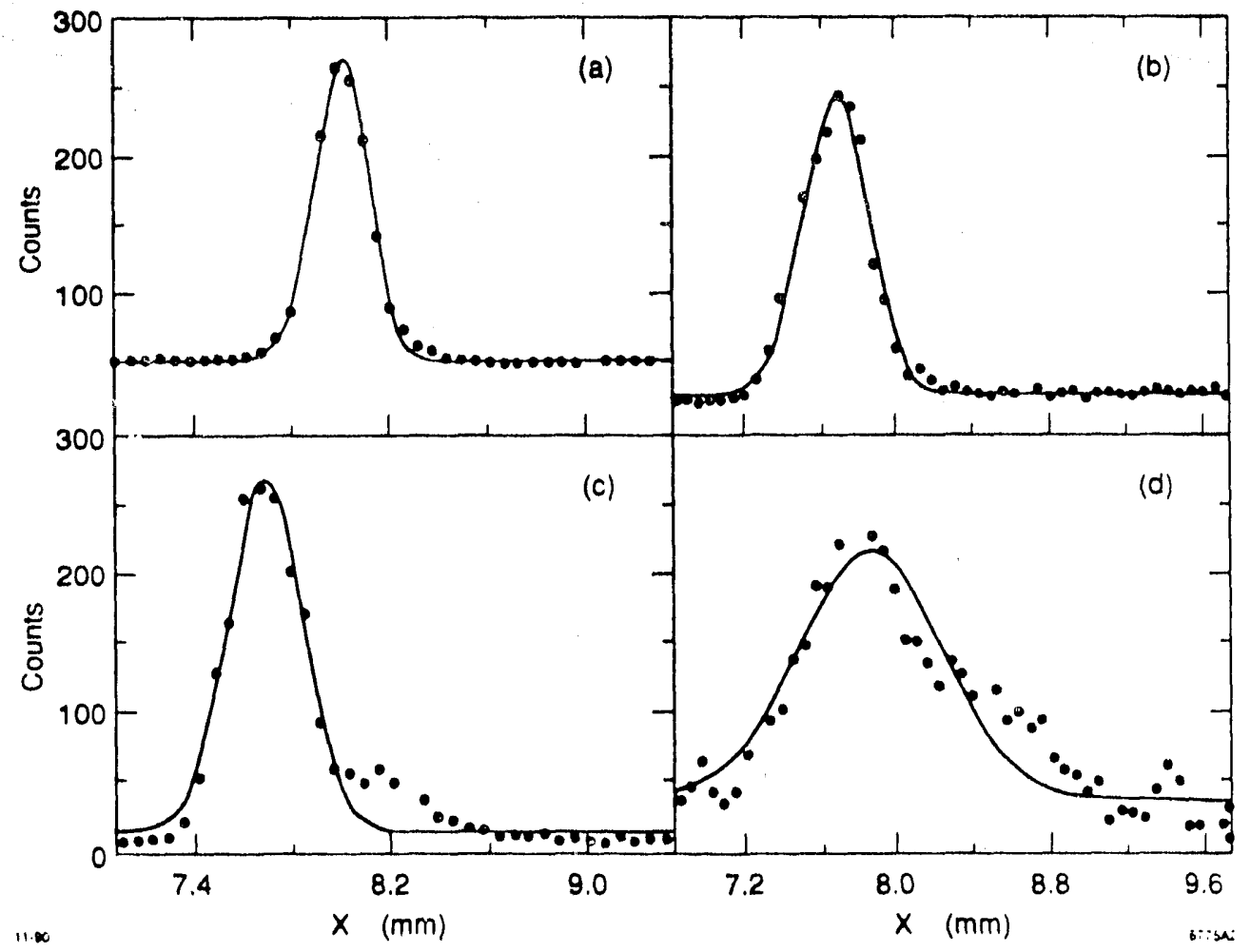

Figure 34 Measured beam profiles ( $47 \mathrm{GeV}$ ) in the SLC for the betatron oscillation in Figure 33 . The projections were taken with four wire scanners at (a) 0 , (b) 22.5 , (c) 90 ., and (d) 112.5 degrees in betatron phase. Note that the induced transverse tail has a particular phase such that the tail does not appear at position (a). The produced profiles have different shapes depending on the betatron phase. Consequently, the transverse tail rotates in phase space at the betatron frequency and makes the beam appear to have a betatron mismatch. 


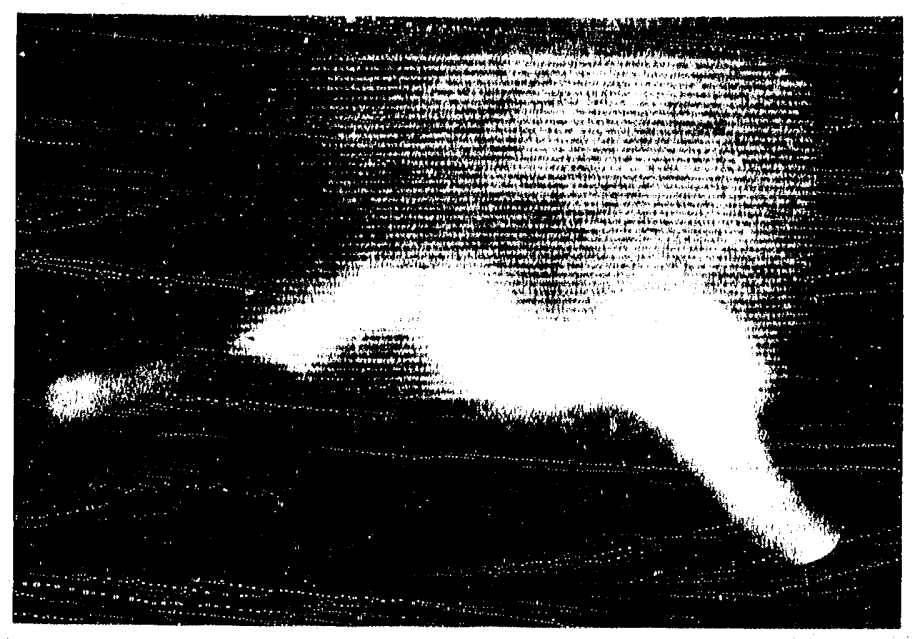

Figure 35 An SLC electron bunch was generated with an unusually long length (of order $2.5 \mathrm{~mm}$ ) and was made to oscillate both horizontally and vertically. The resulting beam was photographed on a profile monitor at the end of the linac. The image displays a "wormlike" correlation of particle positions along the bunch with the bunch head at the lower right. This particle distribution is characteristic of very strong wakefields. The beam length in this picture is $4 \mathrm{~mm}$.

\subsection{Emittance minimization}

After the input parameters are optimized and the trajectory nominally corrected, the beam experiences emittance growth during acceleration because of alignment errors of the accelerator components. This results from the trajectory being steered through misaligned quadrupoles and accelerator structures onto beam position monitors with finite residual offset errors. Consequently, transverse wakefields excite the beam.

Methods to reduce these effects have been theoretically studied [41,42]. It was shown that the addition of appropriate injection errors $\left(\Delta x, \Delta x^{\prime}, \Delta y\right.$, and $\left.\Delta y^{\prime}\right)$ could cancel most of the emittance enlargement. Since the advent of BNS damping, a more global scheme of distributing short range oscillations along the accelerator has been shown to be satisfactory [43]. Oscillations have been added to the SLC linac to test this procedure. Examples of these oscillations are shown in Figure 38. The emittance at full energy was measured as a function of the oscillation amplitude. The results are shown in Figures 39 and 40. A good choice of the amplitude of the short range oscillation in the appropriate linac location significantly reduced the emittance enlargement. Furthermore, the betatron match of the beam was left properly maintained. Combinations of short range $(200 \mathrm{~m})$ oscillations in the SLC were used with two beams during particle physics collisions, the emittances were reduced to the design values at $3 \times 10^{10} \mathrm{e}^{-}$. The required electron trajectories for reducing the emittances are shown in Figure 41. Note that significant trajectory offsets were needed for this optimized case.

At high currents the alignment of the RF structure relative to the quadrupoles and position monitors dominates all other alignment issues. The effects of structure misalignments are schematically shown in Figures 42 and 43. It is a daunting task to align the RF structure at the one meter scale to $100 \mu \mathrm{m}$ or less over many kilometers of linac. Great efforts have been made to develop techniques which will align the structures to these tolerances and to keep them aligned over a long period with minimal effort. 


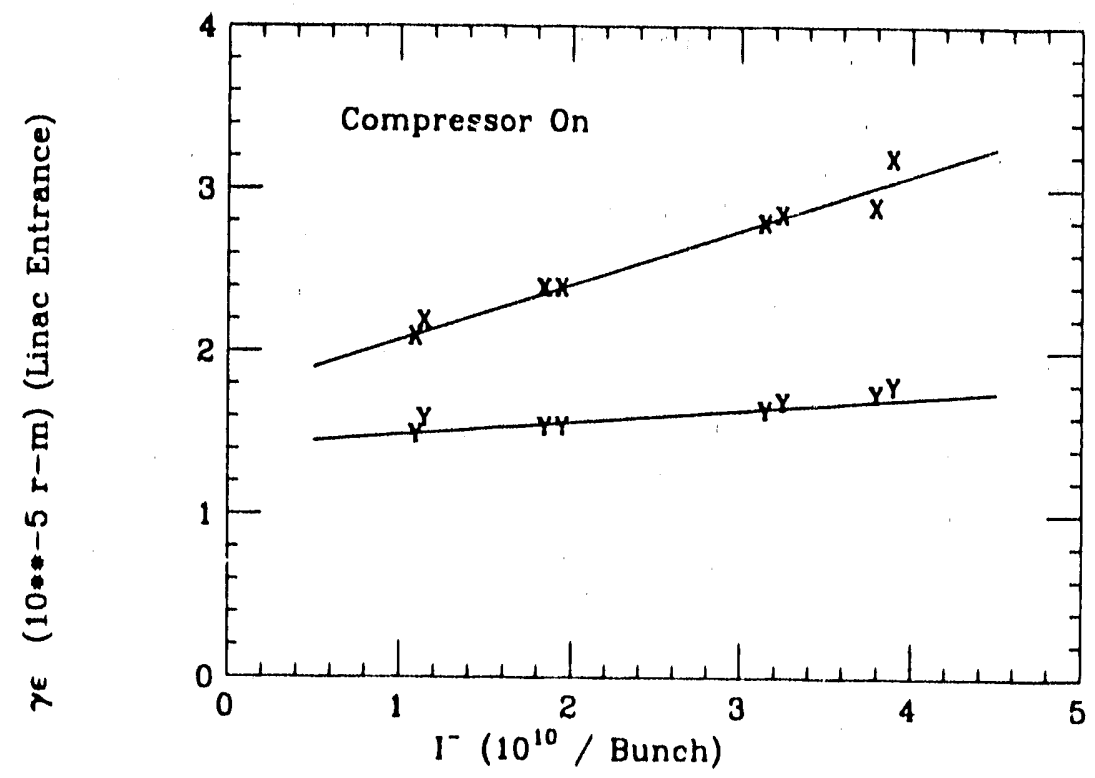

Figure 36 Measured invariant emittances at the entrance to the SLC linac $(1.15 \mathrm{GeV})$ as a function of the beam intensity with the bunch length compressor excited in the standard configuration. The vertical emittance remains constant but the horizontal emittance grows. This growth is associated with first- and second-order chromatic errors in the transport line $\left(\sigma_{E} / E=1 \%\right)$ and the onset of bunch lengthening in the damping ring at about $1 \times 10^{10} \mathrm{e}^{*}$.

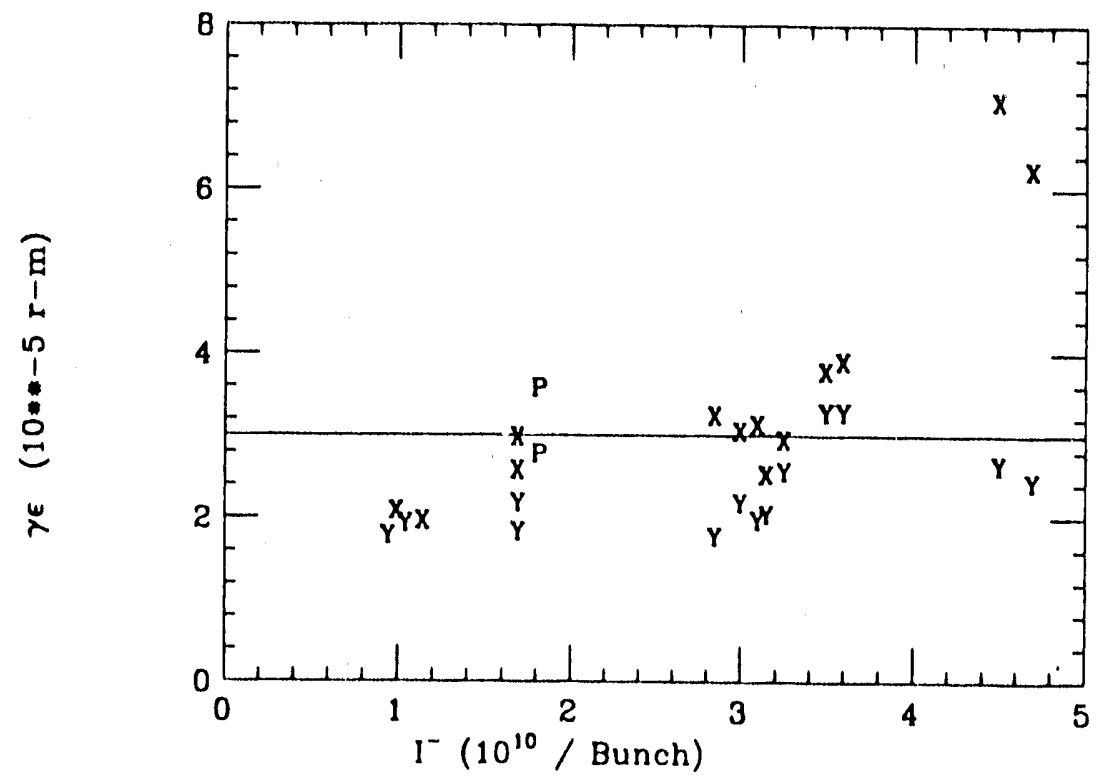

Figure 37 Best measured invariant emittances at the exit of the SLC linac ( $47 \mathrm{GeV})$ as a function of beam current. (The symbol $P$ refers to positrons.) Emittances ( $x$ and $y$ ) at $1 \times 10^{10}$ electrons have been measured at $2 \times 10^{-5} \mathrm{r}-\mathrm{m}$, which is $67 \%$ of the desired value of $3 \times 10^{-5} \mathrm{r}-\mathrm{m}$. At $3 \times 10^{10}$ electrons the allowed emittance values were obtained. Above about $4.0 \times 10^{10} \mathrm{a}$ strong launch jitter is produced from the damping ring system and amplified in the linac which causes larger emittances. Bunch lengthening and wakefields effects in the linac may also contribute. Comparing with Figure 36 , most of the emittance growth to $3 \times 10^{10} \mathrm{e}^{-}$is caused by increases in the injected emittance. 


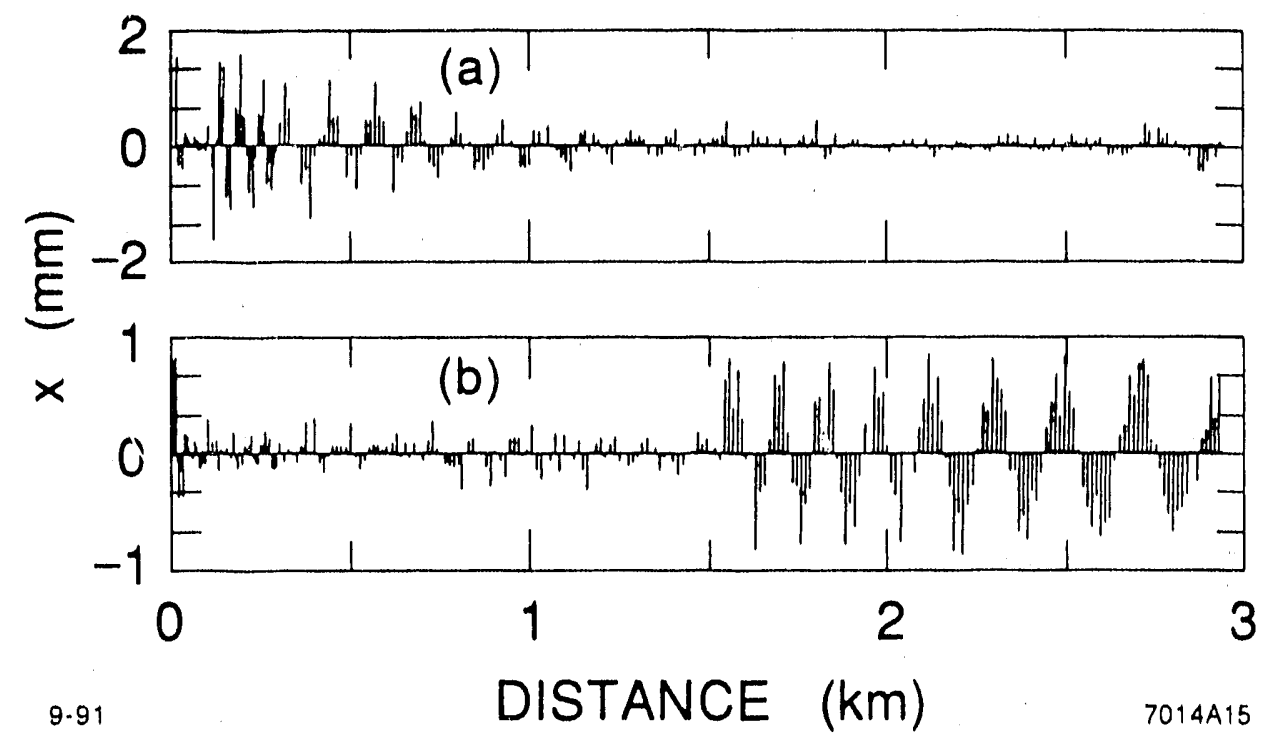

Figure 38 Two induced oscillations in the SLC accelerator used to cancel accumulated wakefield and dispersion errors in the linac, thus reducing the final emittance. See Figures 39 and 40.

Experiments have taken place to study wakefields in misaligned structures [44]. Experimentally, it has been shown that moving structure irises with no other changes can deflect the beam as is shown in Figure 44. These observations have naturally directed efforts towards a possible cure. The component of the structure alignment errors at the betatron spatial frequency is primarily that which drives the beam. Therefore, if a control mechanism can be made to move the structure at that spatial frequency (see Figure 45), then sine and cosine adjustments can be used to cancel the accumulation of alignment errors over reasonably short regions of the accelerator [45]. The alignment tolerances can then be markedly relaxed. This control can also be used to correct for any long term position drift of the accelerator or quadrupole supports due to temperature changes or floor creep in the tunnel.

\subsection{Emittance collimation}

Intense, low emittance beams at the end of a linac are often passed through small collimators to remove unwanted halos or to reduce the effective emittance. If the core of an intense bunch is slightly offaxis in these collimators, the bunch experiences deflecting forces that vary over the bunch depending on the local charge density [46]. A schematic illustration of this effect is shown in Figure 46.

The configuration of the collimation system is important. The desire is to have the sum of the deflections small compared to the beam size. The offset of the beam in the collimator is proportional to the square root of the local beta function $\beta$. The collimator aperture is proportional to the beam size which is proportional to the square root of $\beta$. Thus the kick which is proportional to the ratio of the beam offset in the collimator to the collimator bore is independent of $\beta$. The kick is, however, inversely proportional to the beam energy $\mathrm{E}$. The beam angular divergence is inversely proportional to the square root of the product of the energy and $\beta$. 


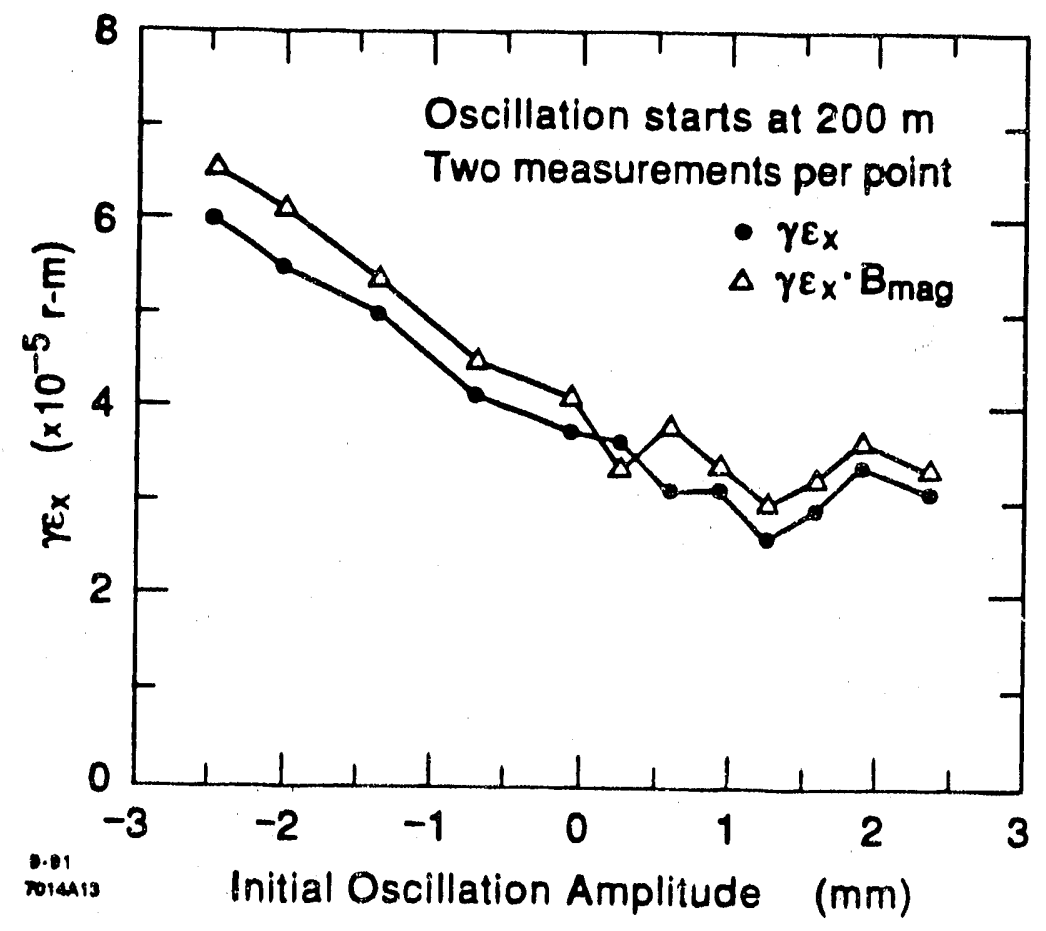

Figure 39 Invariant emittance changes at the end of the linac $(47 \mathrm{GeV})$ as a function of the amplitude of an oscillation starting early in the linac (upper plot in Figure 38). The emittance measurements and the emittance times Bmag measurements track each other very well. Since Bmag is a measure of the expected filamentation from betatron mismatches, the beam at the end of the linac has already filamented. A decrease in the transverse emittance (25\%) is observed with a finite oscillation added to the beam. The error which caused the original emittance enlargement is thus near the beginning of the linac.

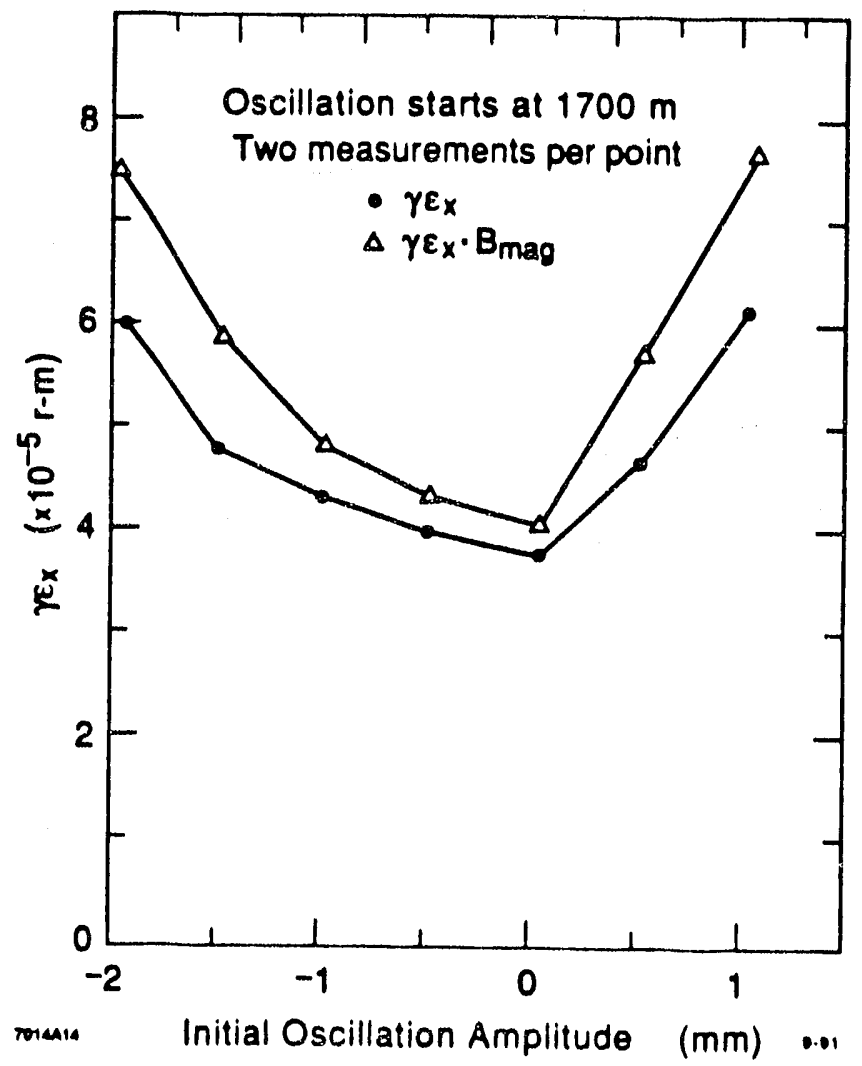

Figure 40 Invariant emittance changes at the end of the linac $(47 \mathrm{GeV})$ as a function of the amplitude of an oscillation starting in the center of the linac (lower plot in Figure 38). There is no reduction of the emittance from this oscillation and, furthermore, a large betatron mismatch has developed signaled by the separation of the curves for $\gamma \varepsilon$ and $\gamma \varepsilon \times$ Bmag. 

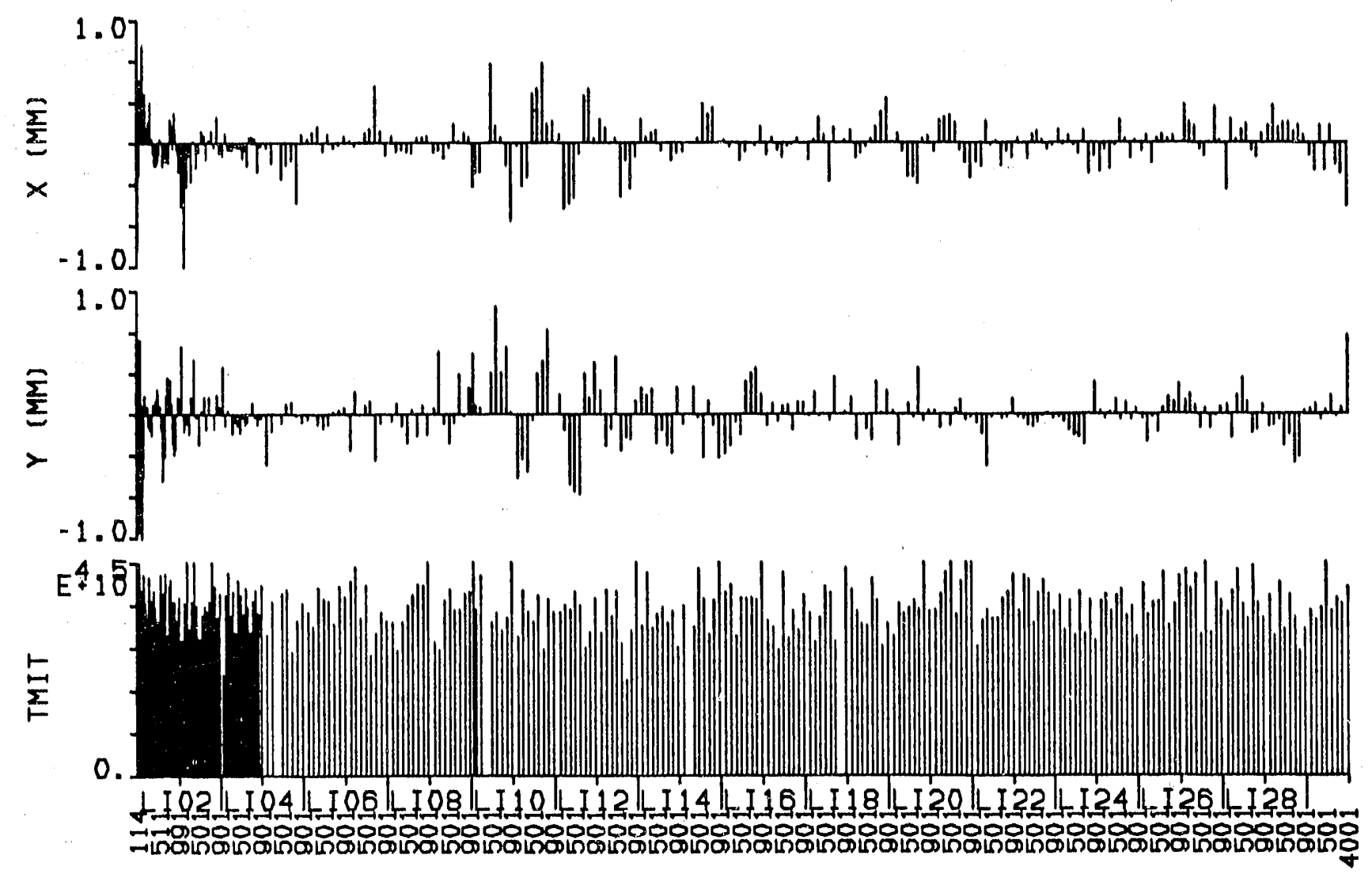

Figure 41 Empirically determined linac trajectory along the $3 \mathrm{~km}$ SLC linac which cancels the errors from the accumulation of dispersion and transverse wakefield errors for a bunch with $3 \times 10^{10}$ electrons. These oscillations lowered the invariant horizontal emittance from 4.5 to $3.0\left(\times 10^{-5} \mathrm{r}-\mathrm{m}\right)$. This trajectory is not unique as other trajectories with similar oscillations can produce the same effect. This non-zero trajectory remained the optimum trajectory during several weeks of collisions.

Therefore,

$$
\frac{\theta_{\text {kick }}}{\theta_{\text {beam }}} \alpha\left[\frac{\beta}{E}\right]^{1 / 2}
$$

where a low $\beta$ and a high $E$ minimize the emittance growth. Unfortunately, a low $\beta$ value leads to small beam sizes and the potential for component radiation damage. For the next linear collider special collimation sections must be designed [47].

\subsection{Multibunch transverse effects}

The acceleration of multiple bunches requires the RF structures to have sufficiently small long range transverse wakefields so that trailing bunches do not experience significant transverse forces. The forces could generate or increase oscillation amplitudes of trailing bunches and ultimately increase their emittances. The solutions to this problem are to space the bunches far enough apart to allow the higher order transverse modes to decohere or to damp the modes with absorbers. The new structures for a future linear collider will likely include a combination of transverse waveguides to damp higher order modes and cell-to-cell detuning to dephase the higher order fields. Furthermore, the captured modes in the structure, cell-to-cell fields, that are driven by long trains of bunches must be detuned to avoid unwanted multiple beam breakup. 


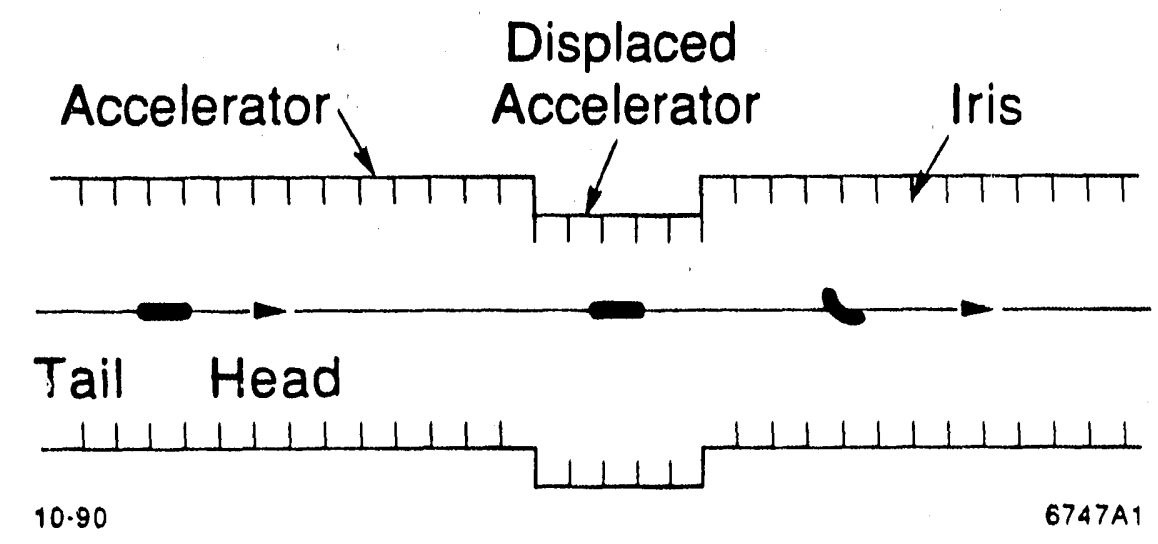

Figure 42 A displaced accelerating structure will induce a transverse offset in the beam from transverse wakefields. The head of the bunch remains on axis but the core continues to drive the tail to ever increasing amplitudes.

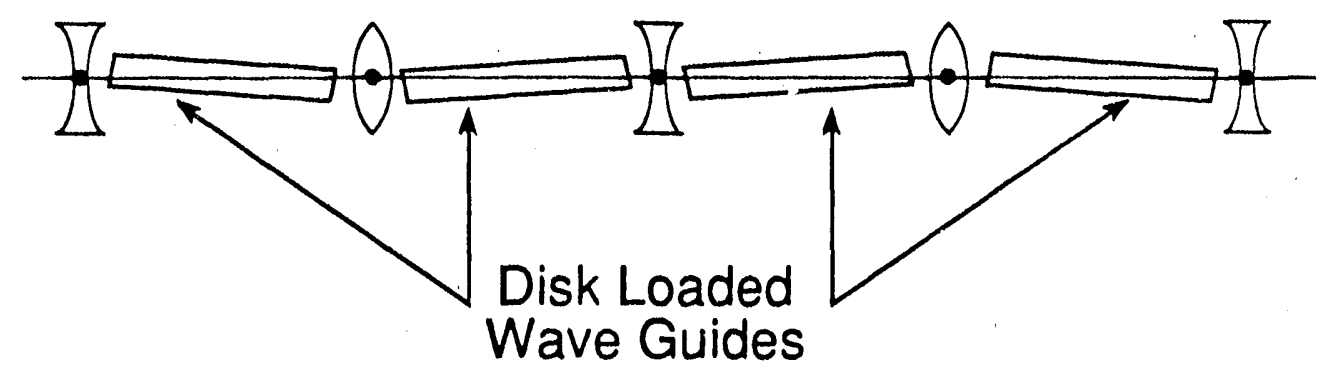

$10-90$

$6748 A 10$

Figure 43 Due to alignment errors, the RF structure displacements in an accelerator, even though small, collectively contribute to the transverse wakefield effects of the beam shape.

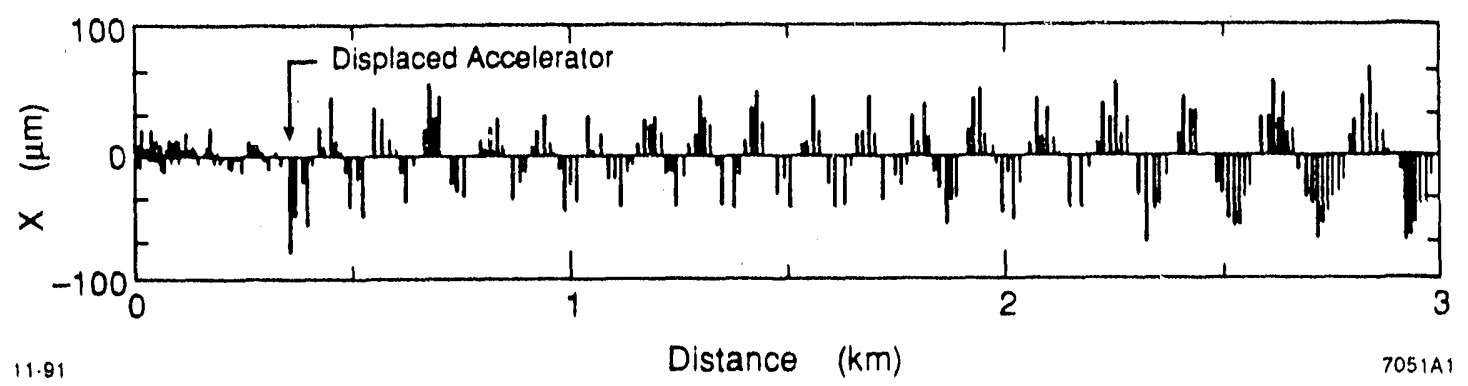

Figure 44 The center of an RF accelerating girder $(12 \mathrm{~m})$ of the SLAC linac was displaced by $1 \mathrm{~mm}$ while leaving all other parameters fixed. The observed transverse oscillation of a beam downstream resulted from the displacement of copper irises only.

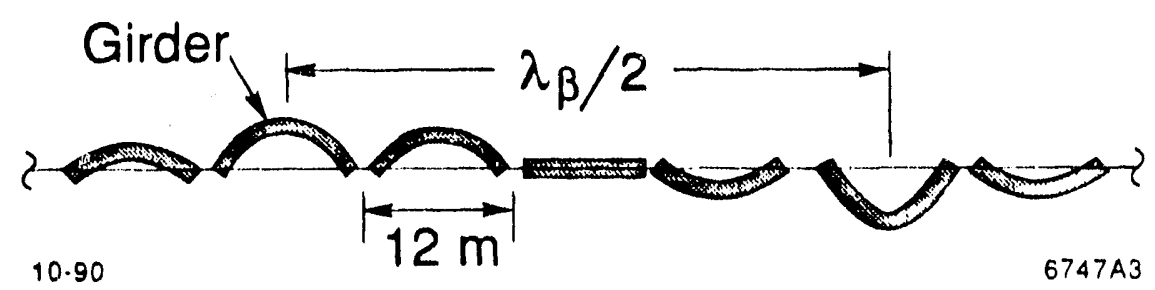

Figure 45 Deliberate distortions of the accelerating structure at the betatron spatial frequency can be used to control random natural misalignments. Quadrupoles are located at the nodes. 


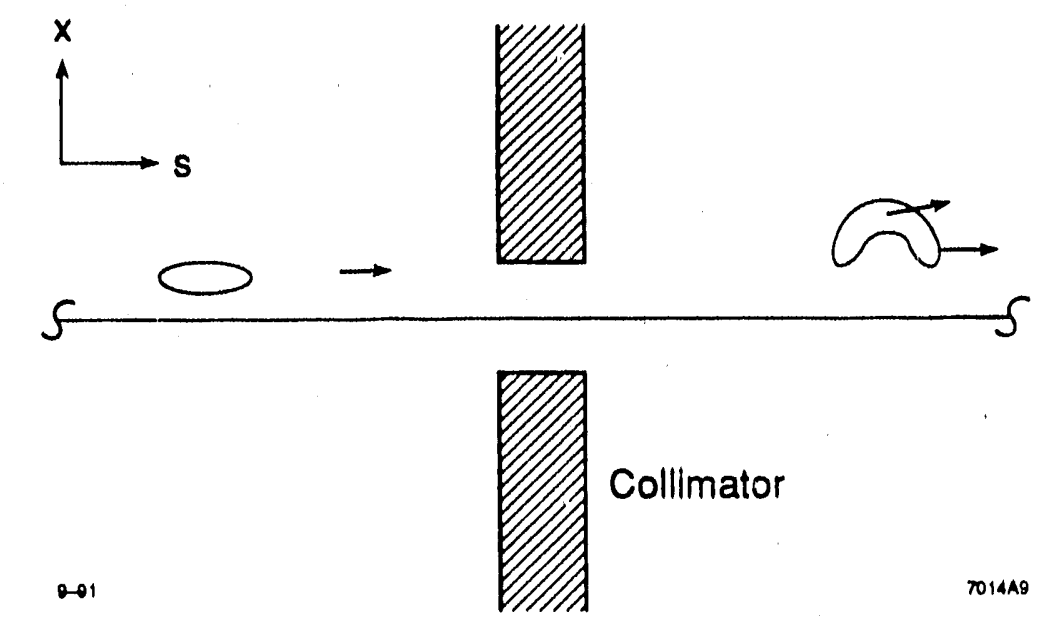

Figure 46 Schematic illustration of the increase in transverse emittance from a close but asymmetric encounter of an intense electron bunch with a beam halo collimator.

Oscillations at high currents have been used to study long range wakefields. Two bunches were made to oscillate along the SLC linac with variable spacings. The results are shown in Figure 47. The trajectory of the second bunch was measured with and without the leading bunch. No noticeable change can be seen for the nominal $60 \mathrm{~ns}$ spacing. Effects were seen, however, with a $10 \mathrm{~ns}$ spacing, as expected [48].

Studies of very closely spaced bunches were also made at the KEK linac as is shown in Figure 48. Their findings were that the deflections are linear in the initial off set, they depend on the charge per bunch, and they depend strongly on the length of the accelerator [49].

\section{Acknowledgments}

The author wishes to thank many people at the Stanford Linear Accelerator Center and from other laboratories around the word for stimulating discussions about high current effects in accelerators. Special thanks are given to the SLC Linac and Emittance Study Groups.

\section{References}

1. C. Pellegrini, 1990 Linear Accelerator Conference, Albuquerque, p. 273 (1990).

2. J. Seeman, Annu. Rev. Nucl. Part. Sci. 1991. 41:389-428.

3. A. Hofmann and H. Winick, privare communication.

4. B. Richter, Part. Accel. 1990. 26: 33-50.

5. $\quad$ R. Palmer, Annu. Rev. Nucl. Prirt. Sci. 1990. 40:529-592.

6. K. Takata and Y. Kimura, Part. Accel. 1990). 26: 87-96.

7. R. Ruth, Proc. of Joint ClER?/US Particle Accelerator School, llilton Head (1990).

8. J. Secman and L. Merminga, 1990 Linear Accelerator Conference, Albuquerque, p. 387 (1990). 

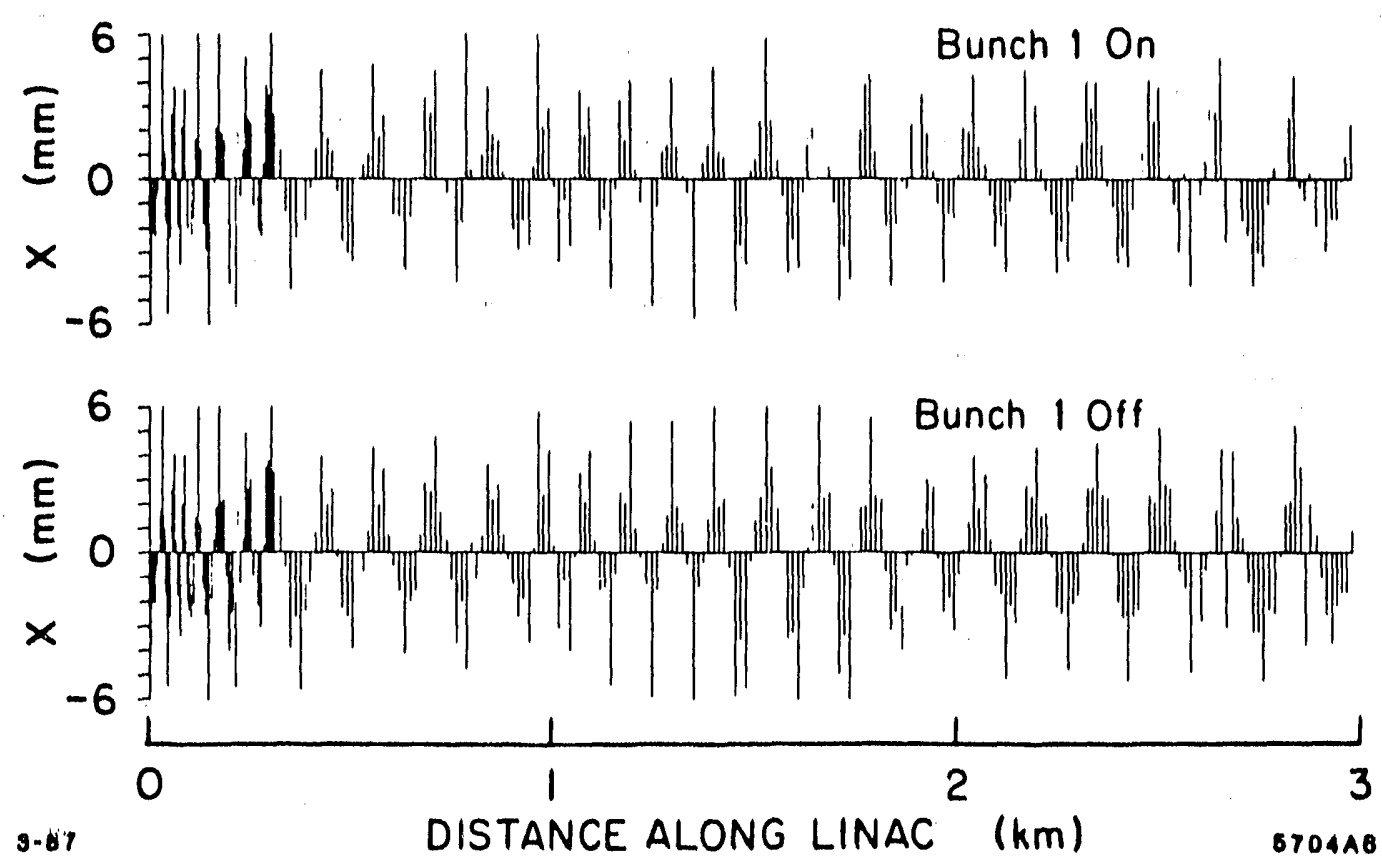

Figure 47 Oscillations of a trailing bunch when the leading bunch in the SLAC linac is present or not. With a spacing of $60 \mathrm{~ns}$ as shown, there is negligible effect. Effects begin to occur with spacings of about $10 \mathrm{~ns}$.

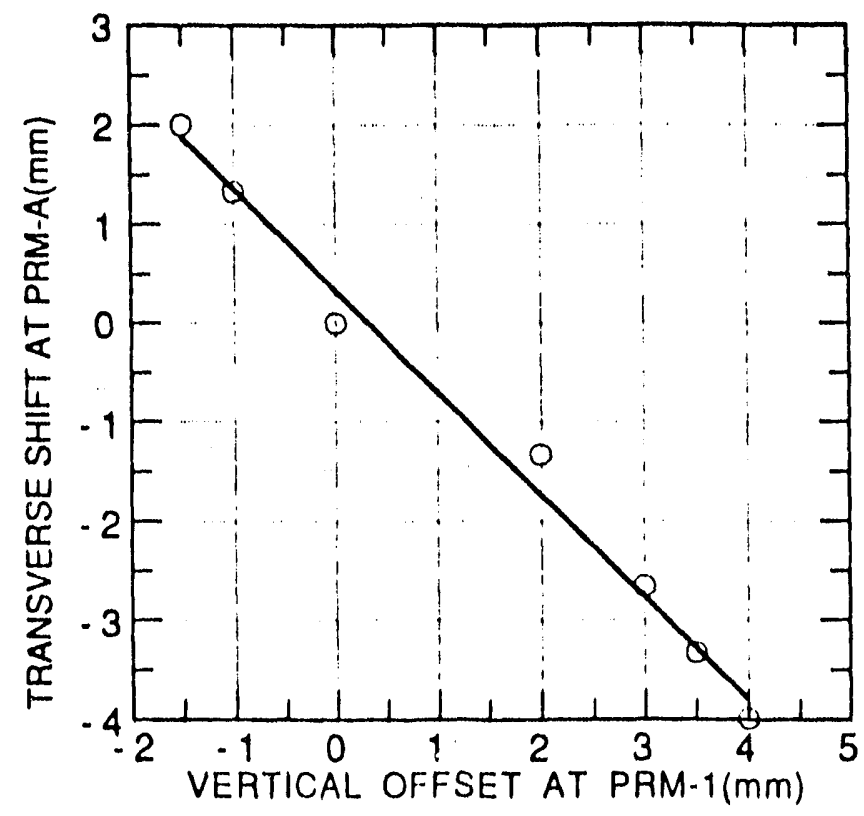

Figure 48 Transverse siift of the beam at the end of the KEK accelerator as a function of initial offset. 
9. K. Bane and P. Wilson, 11 th Int. Conf. on High Energy Accelerators, Birkhauser, p. 592 (1980).

10. D. Warner, 1988 Linear Accelerator Conference, CEBAF, M01-4 (1988).

11. J. Seeman, et al., SLAC-PUB-5438, Stanford (1991).

12. K. Bane, SLAC/AP-80, Stanford (1990).

13. K. Thompson and R. Ruth, IEEE 89CH2669-0, Chicago, p. 792 (1989).

14. Z. D. Farkas, et al, 9th Int. Conf, on High Energy Accel., p. 576 (1974).

15. J. Seeman, et al., IEEE CH2387-9/87/0000, Washington PAC, p. 73 (1987).

16. V. Balakin, et al., 12th Int. Conf. on High Energy Accel., FNAL, p. 119 (1983).

17. H. Henke, 1988 Linear Accelerator Conference, CEBAF, p. 481 (1988).

18. J. Seeman, et al., SLAC-PUB-4968 (to be published).

19. C. Adolphsen, et al., SLAC-PUB-5581, Stanford (1991).

20. V. Balakin, 1988 Workshop on Linear Colliders, SLAC, p. 119 (1988).

21. R. Gluckstern, et al., 1990 Linear Accelerator Conference, Albuquerque, p. 309 (1990).

22. T. Limberg, et al., EPAC, Nice, p. 1506 (1990).

23. J. Seeman, et al., SLAC-PUB-5439, Stanford (1991).

24. T. Himel, et al., SLAC-PUB-5470, Stanford (1991).

25. M. Ross, private communication.

26. J. Seeman, et al., SLC-Reports-249 and 252, Stanford (1991).

27. J. Seeman, SLAC-CN-330, Stanford (1986).

28. J. Seeman, et al., IEEE Trans. Nucl. Sci. 32:2629 (1985).

29. R. Stiening, SLAC-CN-181, Stanford (1982).

30. J. Seeman, IEEE Trans. Nucl. Sci. 87-CH2387-9: p. 1267 (1987).

31. J. Seeman, Part. Accel. 30:7, p. 73 (1990).

32. W. Spence, et al., SLAC-PUB-5276, Stanford (1991).

33. J. Sheppard, SLAC-CN-298, Stanford (1985).

34. J. Seeman, SLAC-CN-330, Stanford (19886)

35. L. Merminga, et al., SLAC-PUB-5514, Stanford (1991).

36. K. Brown and R. Servranckx, SLAC-CN-350, Stanford (1986).

37. J. Seeman, SLAC-PUB-5294, Stanford (1991).

38. J. Seeman, SLAC-PUB-5440, Stanford (1991).

39. K. Bane, SLAC-PUB-5177, Stanford (1990).

40. J. Seeman, et al., SLAC-PUB-5437, Stanford (1991).

41. A. Chao, B. Richter, and C. Yao, Nucl. Inst. and Meth. 178, p. 1 (1980).

42. K. Bane, IEEE Trans. Nucl. Sci. NS-32, No. 5, p. 2389 (1985).

43. J. Seeman, et al., SLAC-PUB-5705, Stanford (1991)

44. J. Seeman, SLAC-PUB-5706, Stanford (1991)

45. J. Seeman, 1990 Linear Accelerator Conference, Albuquerque, p. 387 (1990).

46. K. Bane and P. Morton, 1986 Linear Accelerator Conference, SLAC, p. 490 (1986).

47. L. Merminga, et al., SLAC-PUB-5436, Stanford, (1991).

48. J. Seeman, et al., IEEE CH2387-9/87/000, Washington PAC, p. 73 (1987).

49. Y. Ogawa, T. Shidara, and A. Asami, Phys. Rev. D 43: 1, p. 258 (1991).

\section{DISCLAIMER}

This report was prepared as an account of work sponsored by an agency of the United States Government. Neither the United States Government nor any agency thereof, nor any of their employees, makes any warranty, express or implied, or assumes any legal liability or responsibility for the accuracy, comple'eness, or usefulness of any information, apparatus, product, or process disclosed, or represents that its use would not infringe privately owned rights. Reference herein to any specific commercial product, process, or service by trade name, trademark, manufacturer, or otherwise does not necessarily constitute or imply its endorsement, recommendation, or favoring by the United States Government or any agency thereof. The views and opinions of authors expressed herein do not necessarily state or reflect those of the United States Government or any agency thereof. 

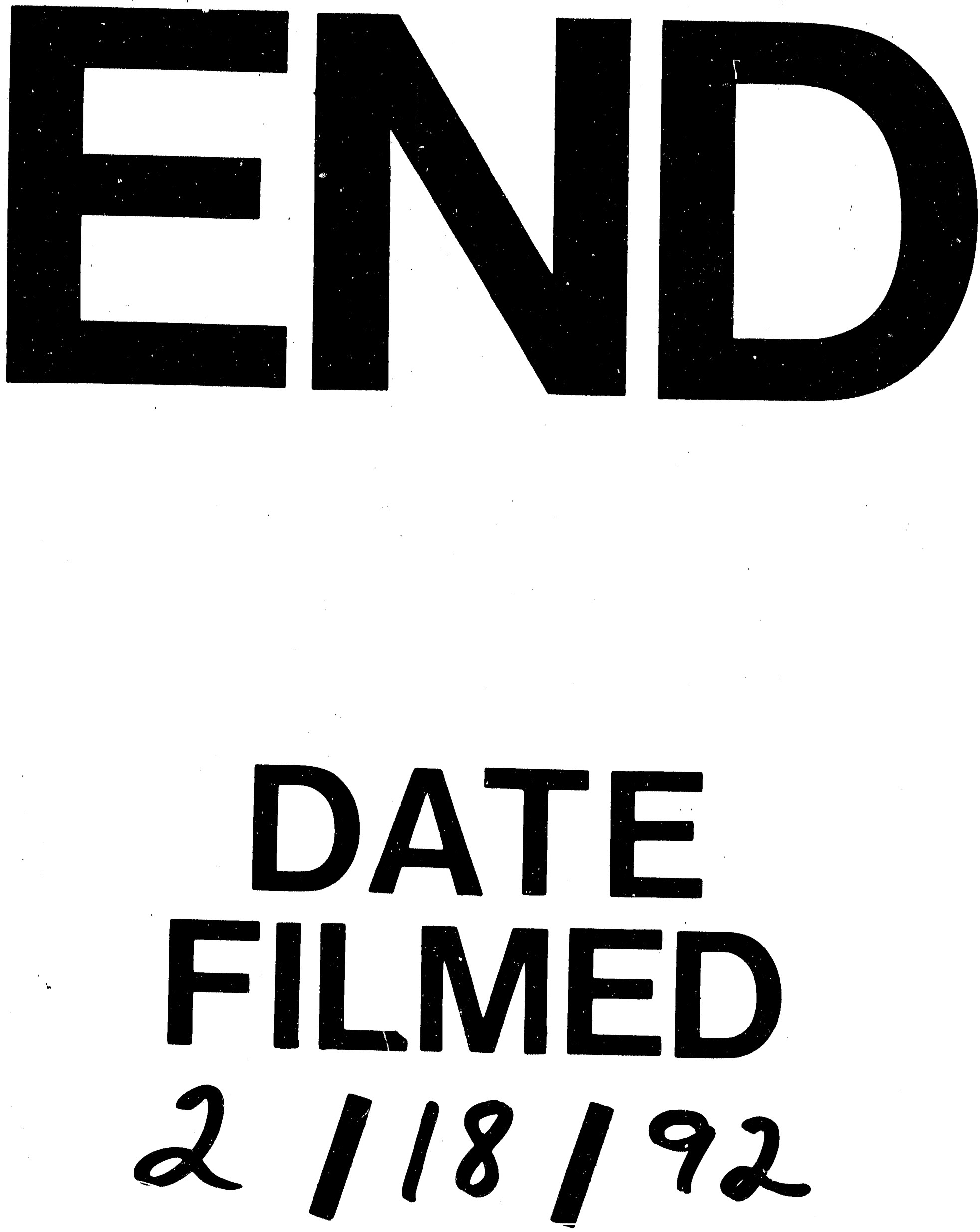